Pacific

Journal of

Mathematics

\title{
RELATIVE FAMILY GROMOV-WITTEN INVARIANTS AND SYMPLECTOMORPHISMS
}

\section{Olguța Buşe}




\title{
RELATIVE FAMILY GROMOV-WITTEN INVARIANTS AND SYMPLECTOMORPHISMS
}

\author{
OlguțA BuşE
}

\begin{abstract}
We study the symplectomorphism groups $G_{\lambda}=\operatorname{Symp}_{0}\left(M, \omega_{\lambda}\right)$ of a closed manifold $M$ equipped with a one-parameter family of symplectic forms $\omega_{\lambda}$ with variable cohomology class. We show that the existence of nontrivial elements in $\pi_{*}\left(\mathscr{A}, \mathscr{A}^{\prime}\right)$, where $\left(\mathscr{A}, \mathscr{A}^{\prime}\right)$ is a suitable pair of spaces of almost complex structures, implies the existence of nontrivial elements in $\pi_{*-i}\left(G_{\lambda}\right)$, for $i=1$ or 2. Suitable parametric Gromov-Witten invariants detect nontrivial elements in $\pi_{*}\left(\mathscr{A}, \mathscr{A}^{\prime}\right)$. By looking at certain resolutions of quotient singularities we investigate the situation $\left(M, \omega_{\lambda}\right)=\left(S^{2} \times S^{2} \times X, \sigma_{F} \oplus \lambda \sigma_{B} \oplus \omega_{\text {arb }}\right)$, with $\left(X, \omega_{\text {arb }}\right)$ an arbitrary symplectic manifold. We find nontrivial elements in higher homotopy groups of $G_{\lambda}^{X}$, for various values of $\lambda$. In particular we show that the fragile elements $w_{\ell}$ found by Abreu and McDuff in $\pi_{4 \ell}\left(G_{\ell+1}^{\mathrm{pt}}\right)$ do not disappear when we consider them in $S^{2} \times S^{2} \times X$.
\end{abstract}

\section{Introduction}

Let $\left(M^{2 n}, \omega\right)$ be a $2 n$-dimensional compact symplectic manifold. The group of symplectomorphisms $\operatorname{Symp}(M, \omega)$ of $M$ is a basic invariant that distinguishes among different symplectic structures on $M$. It is an infinite-dimensional group endowed with a natural $C^{\infty}$ topology.

Two natural questions arise in relation with $\operatorname{Symp}(M, \omega)$ :

(1) What can be said about the topological type of $\operatorname{Symp}(M, \omega)$ ?

(2) How does the topological type change as $\omega$ varies?

Research has been done in this direction by various authors [Abreu 1998; Lê and Ono 2001; McDuff 2000; Seidel 1999; 1997] by using information on $J$ holomorphic curves. We investigate these questions by defining relative parametric GW invariants, which are sensitive to the topology of appropriate spaces of almost

MSC2000: primary 57R17; secondary 53D35, 53D45, 57S05.

Keywords: symplectomorphism group, Gromov-Witten invariant, almost complex structure. 
complex structures. The connection between the spaces of almost complex structures and the symplectomorphism groups is achieved by means of the following fibration, introduced in [Kronheimer 1998] and used in [McDuff 2001]:

$$
\operatorname{Symp}_{0}(M, \omega) \longrightarrow \operatorname{Diff}_{0} M \stackrel{\psi \rightarrow\left(\psi^{-1}\right)^{*} \omega}{\longrightarrow} S_{[\omega]},
$$

where $S_{[\omega]}$ is the space of symplectic forms that can be joined to $\omega$ through a path of cohomologous symplectic forms, $\operatorname{Diff}_{0} M$ is the connected component of the identity inside the group of diffeomorphism and $\operatorname{Symp}_{0}(M, \omega)=\operatorname{Symp}(M, \omega) \cap$ $\operatorname{Diff}_{0} M$. Now consider the space $\mathscr{A}_{[\omega]}$ of almost complex structures that are tamed by some symplectic form in $\mathscr{S}_{[\omega]}$. By [McDuff 2001], $\mathscr{A}_{[\omega]}$ is homotopy equivalent to $\mathscr{S}_{[\omega]}$. This yields the homotopy fibration

$$
\operatorname{Symp}_{0}(M, \omega) \longrightarrow \operatorname{Diff}_{0} M \longrightarrow \mathscr{A}_{[\omega]} .
$$

Our strategy will be to define suitable pairs $\left(\mathscr{A}, \mathscr{A}^{\prime}\right)$ of spaces of almost complex structures, such that information on nontrivial homotopy groups in $\left(\mathscr{A}, \mathscr{A}^{\prime}\right)$ extends to information on $\operatorname{Symp}_{0}(M, \omega)$. We develop relative family $\mathrm{GW}$ invariants that detect such nontrivial elements in $\pi_{*}\left(\mathscr{A}, \mathscr{A}^{\prime}\right)$.

Outline of the methods. In Section 2 we define the invariants as follows: Consider a smooth family of symplectic forms $\left(\omega_{\lambda}\right)_{\lambda \in I}$, where the parameter $\lambda$ varies in the interval $I$ in $\mathbb{R}$ in such a manner that the cohomology classes $\left[\omega_{\lambda}\right]$ may also vary along a line $\mathrm{L}$ inside $H^{2}(M, \mathbb{R})$. For convenience we set $\mathscr{A}_{\lambda}:=\mathscr{A}_{\left[\omega_{\lambda}\right]}$. Consider $D \in H_{2}(M, \mathbb{Z})$ and let $\mathscr{A}_{\lambda, D}^{c} \subset \mathscr{A}_{\lambda}$ be the subspace of those almost complex structures $J$ which do not admit $J$-holomorphic stable maps in the class $D$. Further define $\mathscr{A}_{I}=\bigcup_{\lambda \in I} \mathscr{A}_{\lambda}$, and similarly let $\mathscr{A}_{I, D}^{c}$ be its subset consisting of $\bigcup_{\lambda \in I} \mathscr{A}_{\lambda, D}^{c}$. By a similar argument as in [McDuff 2001], $\mathscr{A}_{I}$ is homotopy equivalent with $\bigcup_{\lambda \in I} \mathscr{S}_{\left[\omega_{\lambda}\right]}$ and hence is connected. We will assume that there is a special almost complex structure $*=J_{\text {basepoint }}$ that belongs to all the spaces $\mathscr{A}_{\lambda, D}^{c}$. Consider a family of almost complex structures $\left(J_{B}, \partial J_{B}, *\right)$ that represent an element in $\pi_{*}\left(\mathscr{A}_{I}, \mathscr{A}_{I, D}^{c}, *\right)$. We will define a homomorphism

$$
\mathrm{PGW}_{D, 0, k}^{M,\left(J_{B}, \partial J_{B}\right)}: \bigoplus_{i=1}^{k} H^{a_{i}}(M, \mathbb{Q})^{k} \rightarrow \mathbb{Q}
$$

by counting $J_{b}$-holomorphic stable maps in class $D$, for all $b \in B$. This is well defined because the class $D$ is never represented as a $J_{b}$-holomorphic stable map if $b \in \partial B$.

Theorem 1.1. (i) The $\mathrm{PGW}_{D, 0, k}^{M,\left(J_{B}, \partial J_{B}\right)}$ are symplectic deformation invariants and depend only on the relative homotopy class of the triple $\left(J_{B}, \partial J_{B}, *\right)$. 
(ii) For a fixed choice of $k, D$ and $\alpha_{i}$ the map $\Theta_{0, k, \alpha_{1}, \ldots, \alpha_{k}}: \pi_{*}\left(\mathscr{A}_{I}, \mathscr{A}_{I, D}^{c}, *\right) \rightarrow \mathbb{Q}$ given by

$$
\Theta_{k, \alpha_{1}, \ldots, \alpha_{k}}\left(\left[\left(J_{B}, \partial J_{B}\right)\right]\right)=\operatorname{PGW}_{D, 0, k}^{M,\left(J_{B}, \partial J_{B}\right)}\left(\alpha_{1}, \ldots, \alpha_{k}\right) .
$$

is a homomorphism.

The reason (i) holds is that the class $D$ is never represented for a $J_{b}$ with $b \in \partial B$.

In Section 3 we will exhibit some examples of nontrivial PGW. There we consider the case where $M=S^{2} \times S^{2} \times X$ for $X$ an arbitrary symplectic manifold and where $\omega=\omega_{\lambda} \oplus \omega_{\text {arb }}$ with $\omega_{\text {arb }}$ an arbitrary symplectic form on $X$ and $\omega_{\lambda}=\sigma_{F} \oplus \lambda \sigma_{B}$, for $\sigma_{F}, \sigma_{B}$ forms (of total area 1) on the fiber and base, and $\lambda \geq 1$. The families $\left(J_{B}, \partial J_{B}\right)$ of almost complex structures are provided for $S^{2} \times S^{2}$ in [Kronheimer 1998] and then further investigated in [Abreu and McDuff 2000]. One has to look at a quotient singularity, $\mathbb{C}^{2} / C_{2 \ell}$, where $C_{2 \ell}$ is the cyclic group of order $2 \ell$ acting diagonally by scalars on $\mathbb{C}^{2}$. The deformation space for the canonical resolution of this singularity provides a $4 \ell-2$ family $\left(J_{B_{\ell}}, \partial J_{B_{\ell}}\right) \in\left(\mathscr{A}_{[\ell, \ell+\epsilon]}, \mathcal{A}_{\ell}\right)$ for which suitable PGWs are nontrivial.

The link between these examples and the corresponding groups of symplectomorphisms will be explained in Section 4. It explain there the extent to which the known homotopy properties (see [Abreu and McDuff 2000]) of $\operatorname{Symp}_{0}\left(S^{2} \times S^{2}, \omega_{\lambda}\right)$ are reflected in the higher homotopy groups of

$$
G_{\lambda}^{X}:=\operatorname{Symp}_{0}\left(S^{2} \times S^{2} \times X, \omega_{\lambda} \oplus \omega_{\mathrm{arb}}\right) .
$$

For every $\left(M, \omega_{\lambda}\right)$ a general symplectic manifold, we set $G_{\lambda}:=\operatorname{Symp}_{0}\left(M, \omega_{\lambda}\right)$.

To be able to address the two questions posed at the beginning, one has to establish first a more precise language in which they make sense. One of the difficulties is that in general there is no direct map $G_{\lambda} \rightarrow G_{\lambda+\epsilon}$. In the particular situation $M=S^{2} \times S^{2} \times \mathrm{pt}$, Abreu and McDuff [2000; McDuff 2001] find natural maps $G_{\lambda}^{\mathrm{pt}} \rightarrow G_{\lambda+\epsilon}^{\mathrm{pt}}$, well defined up to homotopy, and prove:

Theorem 1.2 (Abreu and McDuff). (i) The homotopy type of $G_{\lambda}^{\mathrm{pt}}$ is constant on all the intervals $(\ell-1, \ell]$, with $\ell \geq 2$ a natural number. Moreover, as $\lambda$ passes an integer $\ell \geq 2$, the groups $\pi_{i}\left(G_{\lambda}^{\mathrm{pt}}\right)$, for $i \leq 4 \ell-5$, do not change.

(ii) There is an element $w_{\ell} \in \pi_{4 \ell-4}\left(G_{\lambda}^{\mathrm{pt}}\right) \times \mathbb{Q}$ when $\ell-1<\lambda \leq \ell$ that vanishes for $\lambda>\ell$.

To get around the fact that there is no map $G_{\lambda} \rightarrow G_{\lambda+\epsilon}$ when dealing with a general manifold $M$, we show that for any compact $K \subset G_{\lambda}$ the inclusion $0 \times K \subset$ 
$G_{\lambda}$ extends to a map $h$ that fits into the commutative diagram

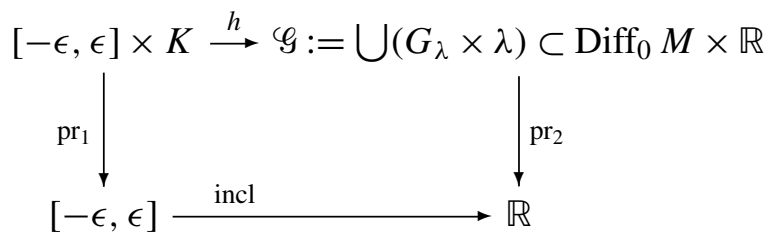

Moreover, for any two such maps $h$ and $h^{\prime}$ coinciding on $0 \times K$, there is, for $\epsilon^{\prime}$ small enough, a homotopy $H:[0,1] \times\left[-\epsilon^{\prime}, \epsilon^{\prime}\right] \times K \rightarrow \mathscr{G}$ between $h$ and $h^{\prime}$ preserving the fibers of the natural projections. Therefore, for any cycle $\rho$ in $G_{\lambda}$, there are extensions $\rho_{\epsilon}$ in $G_{\lambda+\epsilon}$ that, for $\epsilon$ sufficiently small, are unique up to homotopy. Hence they yield well defined elements in $\pi_{*}\left(G_{\lambda+\epsilon}\right)$.

It will therefore make sense to ask what will become of an element $\rho \in \pi_{*}\left(G_{\lambda}\right)$ inside $\pi_{*}\left(G_{\lambda+\epsilon}\right)$, for small $\epsilon$. In this language an element $\theta_{\ell} \in \pi_{*}\left(G_{\ell}\right)$ is called fragile if any extension $\theta_{\ell+\epsilon}$ is null-homotopic in $\pi_{*}\left(G_{\ell+\epsilon}\right)$ for $\epsilon>0$. Also, we say that a family $\eta_{\ell+\epsilon} \in \pi_{*}\left(G_{\ell+\epsilon}\right), 0<\epsilon$ is new if there is no $\eta_{\ell} \in \pi_{*}\left(G_{\ell}\right)$ whose extension is $\eta_{\ell+\epsilon}$. We consider the space $\mathscr{A}_{\ell^{+}}$roughly given by $\mathscr{A}_{\ell^{+}}:=\left(\bigcap_{0<\epsilon<\epsilon_{0}} \mathscr{A}_{\ell+\epsilon}\right) \cup \mathscr{A}_{\ell}$; for the precise definition see (7). We say that an element $\alpha \in \pi_{*}\left(\mathscr{A}_{\ell^{+}}, \mathscr{A}_{\ell}\right)$ is persistent if it has nonzero image under the map $\pi_{*}\left(\mathscr{A}_{\ell^{+}}, \mathscr{A}_{\ell}\right) \rightarrow \pi_{*}\left(\mathscr{A}_{[\ell, \ell+\epsilon]}, \mathscr{A}_{\ell}\right)$.

Our main theorem is the following:

Theorem 1.3. Assume that we have a persistent element $0 \neq \beta_{\ell} \in \pi_{k}\left(\mathscr{A}_{\ell^{+}}, \mathscr{A}_{\ell}, *\right)$. Then we can construct an element $\theta_{\ell} \in \pi_{k-2}\left(G_{\ell}\right)$ such that either

(A) $\theta_{\ell} \in \pi_{k-2}\left(G_{\ell}\right)$ is a nonzero fragile element, or

(B) $\theta_{\ell}=0$ and there is $\epsilon_{\ell}>0$ such that we can construct a family of new elements $0 \neq \eta_{\ell+\epsilon} \in \pi_{k-1}\left(G_{\ell+\epsilon}\right)$, where $0<\epsilon<\epsilon_{\ell}$.

Any fragile element is null-homotopic when viewed inside $\operatorname{Diff}_{0} M$. Our methods do not allow us to decide in general whether or not the image of $\eta_{\ell+\epsilon}$ in $\pi_{k-1} \operatorname{Diff}_{0} M$ is zero.

We show that the hypothesis of the theorem is satisfied when $M=S^{2} \times S^{2} \times X$. We consider $D=A-\ell F$. Since $\left(\sigma_{F} \oplus \ell \sigma_{B} \oplus \omega_{\mathrm{arb}}\right)(A-\ell F)=0$ we get $\mathscr{A}_{\ell} \subset$ $\mathscr{A}_{[\ell, \ell+\epsilon], D}^{c}$. In this situation the $(4 \ell-2)$-dimensional elements $\left(B_{\ell}, \partial B_{\ell}\right)$ obtained in Section 3 are detected as nontrivial in $\pi_{4 \ell-2}\left(\mathscr{A}_{\ell^{+}}, \mathscr{A}_{\ell}\right)$ and are persistent. In fact, in general PGW invariants detect persistent elements. By varying the value of the integer $\ell$ we obtain infinitely many values of $\lambda$ for which higher-order homotopy groups of $G_{\lambda}^{X}$ are nontrivial and we discuss in more detail the stability of the elements $w_{\ell}$ provided by Theorem 1.2 inside $G_{\lambda}^{X}$. We obtain:

Corollary 1.4. For any natural number $\ell \geq 1$, exactly one of the statements below holds. 
(A) We can construct a nonzero fragile element $w_{\ell}^{X} \in \pi_{4 \ell-4}\left(G_{\ell}^{X}\right)$, which can be identified with $w_{\ell} \times$ id.

(B) There exists an $\epsilon_{\ell}>0$ for which we can construct a family of new elements $0 \neq \eta_{\ell+\epsilon}^{X} \in \pi_{4 \ell-3}\left(G_{\ell+\epsilon}^{X}\right), 0<\epsilon<\epsilon_{\ell}$.

In particular this shows that the fragile elements obtained by Abreu and McDuff for $\ell>1$ do not disappear when we consider them inside $S^{2} \times S^{2} \times X$. Either $0 \neq w_{\ell} \times \mathrm{id} \in \pi_{4 \ell-4}\left(G_{\ell}^{X}\right)$ as in (A) or, if $w_{\ell} \times \mathrm{id}=0$ then it yields the associated new $4 \ell-3$ dimensional elements $0 \neq \eta_{\ell+\epsilon}^{X}$ in $\pi_{4 \ell-3}\left(G_{\ell+\epsilon}^{X}\right)$ for small $\epsilon>0$ - this is case (B). For general $\mathrm{X}$ and for $\ell=1$ it is known by work of Lê and Ono that (B) takes place, and moreover that $0 \neq i_{*}\left(\eta_{\ell+\epsilon}\right) \in \pi_{1}\left(\operatorname{Diff}_{0}\left(S^{2} \times S^{2} \times X\right)\right)$, where $i$ is the inclusion of a symplectomorphism group into the diffeomorphism group. Also, for $X=\mathrm{pt}$ and $\ell>1$ we know by work of Abreu and McDuff that (A) takes place.

We don't know of any examples where case (B) takes place and $i_{*}\left(\eta_{\ell+\epsilon}\right)=0 \epsilon$ $\pi_{*}\left(\operatorname{Diff}_{0} M\right)$.

Our method has been inspired by the work of P. Kronheimer, who uses parametric Seiberg-Witten invariants in dimension 4, as well as by [McDuff 2000]. Similar work has been done in this direction in [Lê and Ono 2001]; by looking at related but slightly different parametric GW invariants, these authors get results about $\pi_{k}\left(\operatorname{Symp}_{0}\left(S^{2} \times S^{2} \times X, \omega_{1} \oplus \omega_{\text {arb }}\right)\right)$ when $k=1,3$. In Section 3 we could consider $\mathbb{C}^{2} / C_{2 \ell+1}$ instead and, by carrying out similar arguments, get the same type of results for $\mathbb{C} P^{2} \# \overline{\mathbb{C} P^{2}} \times X$.

\section{Relative parametric GW invariants}

General setting. Consider a compact manifold $B$ with boundary and a smooth map $i:(B, \partial B) \rightarrow\left(\mathscr{A}_{I}, \mathscr{A}_{I, D}^{c}\right)$. Although the invariants can be defined in this generality, for the applications we have in mind we will consider $B$ to be an $n$-ball such that $i$ represents a relative homotopy class in $\pi_{*}\left(\mathscr{A}_{I}, \mathscr{A}_{I, D}^{c}, *\right)$. We will often write $J_{b}:=i(b)$ and $J_{B}=\operatorname{im} i$, and refer to $\operatorname{im} B$ in $\mathscr{A}_{I}$ as $J_{B}$. Consider also a smooth family of symplectic forms $\omega_{B}:=\left(\omega_{b}\right)_{b \in B}$ where $\omega_{b}$ tames $J_{b}$. The $\omega_{b}$ need not be cohomologous, since the taming condition is an open condition. Our goal here is to show how we can define parametric GW invariants relative to the boundary $\partial J_{B}$ of $J_{B}$, invariants that count $J_{b}$-holomorphic maps for some $b \in B$. These will not depend either on deformations of the family $\omega_{B}$ or on the representative $\left(J_{B}, \partial J_{B}\right)$ of a relative homotopy class in $\left(\mathscr{A}_{I}, \mathscr{A}_{I, D}^{c}\right)$.

Consider the space $\widetilde{M}_{0, k}^{*}\left(M, D,\left(J_{B}, \partial J_{B}\right)\right)$ of tuples $\left(b, f, x_{1}, \ldots, x_{k}\right)$, where $f: S^{2} \rightarrow M$ is a simple ${ }^{1} J_{b}$-holomorphic map in class $D$, for some $b \in B$, and the

\footnotetext{
${ }^{1}$ We say that $f: \Sigma \rightarrow M$ is simple if it is not the composite of a holomorphic branched covering map $(\Sigma, j) \rightarrow\left(\Sigma^{\prime}, j^{\prime}\right)$ of degree greater than 1 with a J-holomorphic map $\Sigma^{\prime} \rightarrow M$.
} 
$x_{i}$ are pairwise distinct points on $S^{2}$. We will consider

$$
\mathcal{M}_{0, k}^{*}\left(M, D,\left(J_{B}, \partial J_{B}\right)\right)=\widetilde{M}_{0, k}^{*}\left(M, D,\left(J_{B}, \partial J_{B}\right)\right) / G,
$$

where $G=\operatorname{PSL}(2, \mathbb{C})$ acts on the moduli space by reparametrizations of the domain. Denote the elements of $\mu_{0, k}^{*}\left(M, D,\left(J_{B}, \partial J_{B}\right)\right)$ by $\left[b, f, x_{1}, \ldots, x_{k}\right]$.

In the best scenario, for a good choice of $\left(J_{B}, \partial J_{B}\right)$,

(P1) $\widetilde{M}_{0, k}^{*}\left(M, D,\left(J_{B}, \partial J_{B}\right)\right)$ is a manifold of dimension $2 n+2 c_{1}(D)+2 k+\operatorname{dim} B$, and

(P2) $\mathcal{M}_{0, k}^{*}:=\mathcal{M}_{0, k}^{*}\left(M, D,\left(J_{B}, \partial J_{B}\right)\right)$ is compact.

Then the image of the map

$$
e v: M_{0, k}^{*}\left(M, D,\left(J_{B}, \partial J_{B}\right)\right) \rightarrow M^{k}
$$

with $e v\left(\left[b, f, x_{1}, \ldots, x_{k}\right]\right):=\left(f\left(x_{1}\right), \ldots, f\left(x_{k}\right)\right)$ will provide a cycle $e v_{*}\left(\mathcal{M}_{0, k}^{*}\right)$ in $M^{k}$ which, by intersection with homology classes of complementary dimension in $M^{k}$, gives the parametric Gromov-Witten invariants.

Definition and properties of $P G W$. As the regularity discussion below will make clear, condition (P1) can always be achieved by the Sard-Smale theorem. However, even in situations when (P1) holds, (P2) is seldom true; the compactification $\bar{M}_{0, k}\left(M, D,\left(J_{B}, \partial J_{B}\right)\right)$ of $\mathcal{M}_{0, k}^{*}\left(M, D,\left(J_{B}, \partial J_{B}\right)\right)$ contains both stable $J$ holomorphic maps ${ }^{2}$ and nonsimple curves, which we sometimes call multiple cover curves. These nonsimple curves could potentially produce strata of high dimension in the compactification $\bar{M}_{0, k}\left(M, D,\left(J_{B}, \partial J_{B}\right)\right)$, and hence this space would not necessarily carry a fundamental class.

In the situation $B=\mathrm{pt}$, there are various procedures [Li and Tian 1998; Ruan 1999; Fukaya and Ono 1999] to build up a theory that would provide $a$ virtual moduli cycle, that is, an object carrying a fundamental class required for the definition of the invariants.

Roughly speaking, locally one needs to consider here all the stable holomorphic maps as well as small perturbations of them. There are then various procedures to pass to a global object with the required properties. These go through without essential changes if one considers parameter spaces with no boundary; see [Bryan and Leung 2000; Ruan 1999].

In our situation we need to make sure that the boundary causes no problem. In what follows denote by $\left[f, \Sigma, x_{1}, \ldots, x_{k}\right]$ the equivalence class of a stable map $\left(f, \Sigma, x_{1}, \ldots, x_{k}\right)$, where two maps are equivalent if they differ by an automorphism of the domain. The elements of $\bar{M}_{0, k}\left(M, D,\left(J_{B}, \partial J_{B}\right)\right)$ consist of such

\footnotetext{
${ }^{2}$ These are rational maps $f:\left(\Sigma, x_{1}, \ldots, x_{k}\right) \rightarrow M$ with the most normal crossing singularities and no infinitesimal automorphisms; see [Li and Tian 1998; Bryan and Leung 2000] for details.
} 
equivalence classes. The next result states that if we consider an appropriately small open neighborhood of $\bar{M}_{0, k}\left(M, D,\left(J_{B}, \partial J_{B}\right)\right)$ consisting of almost holomorphic stable maps, its projection onto $J_{B}$ stays away from $\partial J_{B}$.

Lemma 2.1. For any compact set $J_{B} \in \mathscr{A}_{I}$ such that $\partial J_{B} \subset \mathscr{A}_{I, D}^{c}$, there exist $\delta>0$ and $\epsilon(\delta)>0$ for which there is no stable map $\left(f, \Sigma, x_{1}, \ldots, x_{k}\right)$ such that $\bar{\partial}_{J} f=v$, when $d\left(J, \partial J_{B}\right)<\delta$ and $v \in L^{p}\left(\Lambda^{0,1} \otimes_{J} f^{*} T M\right)$ with $|v| \leq \epsilon(\delta)$.

Proof. We will prove this by assuming the opposite. Assume we have sequences $J_{i}, v_{i}$ and $f_{i}$ such that $d\left(J_{i}, \partial J_{B}\right) \rightarrow 0,\left|v_{i}\right|=\epsilon_{i} \rightarrow 0$ and each $f_{i}$ is a stable map in class $D$ with the property that $\bar{\partial}_{J_{i}} f_{i}=v_{i}$. Since $J_{B}$ is compact we find a convergent subsequence $J_{i}$ whose limit $J_{\infty}$ is in $\partial J_{B}$. But by the Gromov compactness theorem there is a subsequence of $f_{i}$ converging to a $J_{\infty}$ stable holomorphic map in class $D$. This contradicts the fact that $J_{\infty} \in \partial J_{B} \subset \mathscr{A}_{I, D}^{c}$.

With this lemma one shows, as in [Li and Tian 1998], that every moduli space $\bar{M}_{0, k}\left(M, D,\left(J_{B}, \partial J_{B}\right)\right)$ carries a virtual fundamental cycle

$$
[\mathcal{M}]^{\mathrm{vir}}:=\left[\bar{M}_{0, k}\left(M, D,\left(J_{B}, \partial J_{B}\right)\right)\right]^{\mathrm{vir}}
$$

of degree $r=2 c_{1}(D)+2 k+2 n-6+\operatorname{dim} B$.

Moreover, if we take two homotopic maps $i:(B, \partial B, *) \rightarrow\left(\mathscr{A}_{I}, \mathscr{A}_{I, D}^{c}, *\right)$ and $i^{\prime}:\left(B^{\prime}, \partial B^{\prime}, *\right) \rightarrow\left(\mathscr{A}_{I}, \mathscr{A}_{I, D}^{c}, *\right)$ representing the same element in $\pi_{*}\left(\mathscr{A}^{\prime}, \mathscr{A}_{D}^{c}, *\right)$, then the corresponding fundamental cycles given by $\left[\bar{M}_{0, k}\left(M, D,\left(J_{B}, \partial J_{B}\right)\right)\right]^{\mathrm{vir}}$ and $\left[\bar{M}_{0, k}\left(M, D,\left(J_{B^{\prime}}, \partial J_{B^{\prime}}\right)\right)\right]^{\text {vir }}$ are oriented cobordant and hence the virtual fundamental class $[M]^{\mathrm{vir}}$ is independent of the choice of $\left(J_{B}, *\right)$ within the same class in $\pi_{*}\left(\mathscr{A}_{I}, \mathscr{A}_{I, D}^{c}, *\right)$. Note that $[\mathcal{M}]^{\mathrm{vir}}$ is also invariant under symplectic deformation of the family of taming symplectic forms $\left(\omega_{b}\right)_{b \in B}$. We denote by $\overline{\mathscr{F}}_{D}(M, 0, k)$ the space of all equivalences classes of stable maps $\left[f, \Sigma, x_{1}, \ldots, x_{k}\right]$ with total homology $D$. To define relative parametric Gromov-Witten invariants we consider $e v_{i}: B \times \overline{\mathscr{F}}_{D}(M, 0, k) \rightarrow M$ given by

$$
e v_{i}\left(b,\left[f, \Sigma, x_{1}, \ldots, x_{k}\right]\right)=f\left(x_{i}\right) .
$$

We then can define

$$
\mathrm{PGW}_{D, 0, k}^{M,\left(J_{B}, \partial J_{B}\right)}: \bigoplus_{i=1}^{k} H^{a_{i}}(M, \mathbb{Q})^{k} \rightarrow \mathbb{Q}
$$

by

$$
\mathrm{PGW}_{D, 0, k}^{M,\left(J_{B}, \partial J_{B}\right)}\left(\alpha_{1}, \ldots, \alpha_{k}\right)=e v_{1}^{*}\left(\alpha_{1}\right) \wedge \cdots \wedge e v_{k}^{*}\left(\alpha_{k}\right)[M]^{\mathrm{vir}}
$$

which are zero unless

$$
\sum_{i=1}^{k} a_{i}=2 c_{1}(D)+2 k+2 n-6+\operatorname{dim} B .
$$


Changing the orientation of $B$ just changes the sign of the invariant.

Theorem 2.2. (i) The invariants $\mathrm{PGW}_{D, 0, k}^{M,\left(J_{B}, \partial J_{B}\right)}$ are symplectic deformation invariants and depend only on the relative homotopy class of $\left(J_{B}, \partial J_{B}\right)$.

(ii) For a fixed choice of $k, D$ and $\alpha_{i}$ the map $\Theta_{0, k, \alpha_{1}, \ldots, \alpha_{k}}: \pi_{*}\left(\mathscr{A}_{I}, \mathscr{A}_{I, D}^{c}, *\right) \rightarrow \mathbb{Q}$ given by

$$
\Theta_{k, \alpha_{1}, \ldots, \alpha_{k}}\left(\left[\left(J_{B}, \partial J_{B}\right)\right]\right)=\mathrm{PGW}_{D, 0, k}^{M,\left(J_{B}, \partial J_{B}\right)}\left(\alpha_{1}, \ldots, \alpha_{k}\right)
$$

is a group homomorphism. ${ }^{3}$

Proof. Point (i) and the well definedness of $\Theta$ follow from the properties of PGW listed above. To show that $\Theta$ is a homeomorphism, choose $\left(B_{1}, \partial B_{1}, *\right)$, and $\left(B_{2}, \partial B_{2}, *\right)$ representing two maps from the standard $n$-ball with boundary to $\left(\mathscr{A}_{I}, \mathscr{A}_{I, D}^{c}, *\right)$, giving two elements $\beta_{1}$ and $\beta_{2}$ inside $\pi_{*}\left(A_{A}, \mathscr{A}_{D}^{c} *\right)$. We choose them in such a way that by their concatenation we represent the element $\beta_{1}+\beta_{2}$ by a map $j:(B, \partial B, *) \rightarrow\left(\mathscr{A}_{I}, \mathscr{A}_{I, D}^{c}, *\right)$ with $j(B \backslash \partial B)=\left(B_{1} \backslash \partial B_{1}\right) \cup\left(B_{2} \backslash \partial B_{2}\right)$, so that $j^{-1}\left(\mathscr{A}_{I} \backslash \mathscr{A}_{I, D}^{c}\right)$ is included in the disjoint union of two open subdiscs in $B$. Then the new virtual cycle corresponding to the classes $\beta_{1}+\beta_{2}$ is a disjoint union of the virtual neighborhoods corresponding to $\beta_{1}$ and $\beta_{2}$. But this implies that the parametric invariants corresponding to the new class $\beta_{1}+\beta_{2}$ are the sum of the PGW corresponding to $\beta_{1}$ and $\beta_{2}$. Therefore $\Theta$ is a homomorpism.

More on the relation between PGW and almost complex structures. We will now see that PGW detects only certain kinds of relative homotopy classes of almost complex structures. As before, we write $\mathscr{A}_{\lambda}=\mathscr{A}_{\omega_{\lambda}}$. Set

$$
\mathscr{A}_{\ell^{+}}=\left\{J \mid \text { there is } \epsilon_{J}>0 \text { such that } J \in \mathscr{A}_{\ell+\epsilon} \text { for all } 0<\epsilon<\epsilon_{J}\right\}
$$

Then $\mathscr{A}_{\ell} \subset \mathscr{A}_{\ell^{+}}$by Lemma 4.1 below. Note that $\mathscr{A}_{\ell^{+}}$may not be connected, but $\mathscr{A}_{\ell}$ is and we will consider our basepoint $*=J_{\text {basepoint }} \in \mathscr{A}_{\ell}$.

Definition 2.3. Consider a nontrivial element $\beta_{\ell} \in \pi_{*}\left(\mathscr{A}_{\ell^{+}}, \mathscr{A}_{\ell}\right)$. We say that $\beta_{\ell}$ is persistent if its image under the natural morphism

$$
i_{*}: \pi_{*}\left(\mathscr{A}_{\ell^{+}}, \mathscr{A}_{\ell}, *\right) \rightarrow \pi_{*}\left(\mathscr{A}_{[\ell, \ell+\epsilon]}, \mathscr{A}_{\ell}, *\right)
$$

is nonzero for any arbitrary small $\epsilon$.

Proposition 2.4. Assume there is an $\ell$ such that no $J$ in $\mathscr{A}_{\ell}$ admits $J$-holomorphic stable maps in class $D$. Consider an element $0 \neq \beta_{\ell} \in \pi_{*}\left(\mathscr{A}_{\ell^{+}}, \mathcal{A}_{\ell}, *\right)$ obtained by counting nontrivial parametric Gromov-Witten invariants in class $D$. Then $\beta_{\ell}$ is a persistent element.

Proof. The proof follows directly from Theorem 2.2.

\footnotetext{
${ }^{3}$ Except in the case of $\pi_{1}\left(\mathscr{A}_{I}, \mathscr{A}_{I, D}^{c}, *\right)$, which is not a group.
} 
Computability of PGW. We will now get back to the two conditions we posed at the beginning of the section, sufficient to imply that the image of the map (5) is a cycle. Below we will provide sufficient hypotheses on the parameter space $\left(J_{B}, \partial J_{B}, *\right)$ and on the class $D$ such that (P1) and (P2) are satisfied, as well as a criterion for how to check one of the hypothesis. It will follow for such a family $\left(J_{B}, \partial J_{B}, *\right)$ the invariants PGW defined above are integer-valued and can be obtained by intersecting the image of the cycle $e v_{*}\left(\overline{\mathcal{M}}_{0, k}^{*}\left(M, D,\left(J_{B}, \partial J_{B}\right)\right)\right)$ with the classes $\left(P D\left(\alpha_{1}\right), \ldots, P D\left(\alpha_{k}\right)\right)$ in $H_{*}(M)^{k}$. Moreover, they can be obtained by counting the number of $J_{b}$-holomorphic maps in class $D$ with $k$ marked points which intersect generic cycles representing $\left(P D\left(\alpha_{1}\right), \ldots, P D\left(\alpha_{k}\right)\right)$ in $f\left(z_{i}\right)$.

Parametric regularity. We now show that $D$-parametric regular families $\left(J_{B}, \partial J_{B}\right)$ are ones for which (P1) is satisfied. We begin by explaining what D-parametric regularity is and contrasting it with the usual D-regularity for $J$ (see [McDuff and Salamon 1994]). For this we need the following facts.

Let $\mathscr{X}=\operatorname{Map}(\Sigma, M ; D)$ be the space of somewhere injective 4 smooth maps $f: \Sigma \rightarrow M$ representing class D. This is an infinite-dimensional manifold with $T_{f} \mathscr{X}=C^{\infty}\left(f^{*} T M\right)$. We will next consider the following generalized vector bundle $\mathscr{E} \rightarrow B \times \mathscr{X}$, whose fiber at $(b, f)$ is the space $\mathscr{E}_{b, f}=\Omega_{J_{b}}^{0,1}\left(f^{*} T M\right)$ of smooth $J_{b}$ antilinear forms with values in $f^{*} T M$. In this vector bundle we consider a section $\Phi: B \times \mathscr{X} \rightarrow \mathscr{E}$, given by

$$
\Phi(b, f)=\frac{1}{2}\left(d f+J_{b} \circ d f \circ j\right) .
$$

The zeros of $\Phi$ are precisely the $J_{b}$-holomorphic maps and thus the moduli space

$$
\widetilde{M}_{0,0}^{*}\left(M, D,\left(J_{B}, \partial J_{B}\right)\right)=\Phi^{-1}(0),
$$

is the intersection of im $\Phi$ with the zero section of the bundle. Since we would like $\widetilde{M}_{0, k}^{*}\left(M, D,\left(J_{B}, \partial J_{B}\right)\right)$ to be a manifold, we require that $\Phi$ be transversal to the zero section. This means that the image of $d \Phi(b, f)$ is complementary to the tangent space $T_{b} B \oplus T_{f} \mathscr{L}$ of the zero section. But for any $f$ which is $J_{b^{-}}$ holomorphic, $d \Phi$ is given by

$$
d \Phi(b, f): T_{b} B \oplus C^{\infty}\left(f^{*} T M\right) \longrightarrow T_{b} B \oplus T_{f} \mathscr{X} \oplus \mathscr{E}_{b, f} .
$$

If we now consider the projection onto the vertical space of the bundle,

$$
\operatorname{proj}_{2}: T_{b} B \oplus T_{f} \mathscr{L} \oplus \mathscr{E}_{b, f} \longrightarrow \mathscr{E}_{b, f},
$$

\footnotetext{
${ }^{4}$ We say that a map $f: \sigma \rightarrow M$ is somewhere injective if $d f(z) \neq 0$ and $f^{-1}(f(z))=z$ for some $z \in \Sigma$. A simple J-holomorphic map is somewhere injective; see [McDuff and Salamon 1994].
} 
the transversality mentioned above translates into the fact that

$$
\operatorname{proj}_{2} \circ d \Phi(b, f): T_{b} B \oplus C^{\infty}\left(f^{*} T M\right) \longrightarrow \Omega_{J_{b}}^{0,1}\left(\Sigma, f^{*} T M\right)
$$

is onto. We introduce the notation $D \Phi(b, f)=\operatorname{proj}_{2} \circ d \Phi(b, f)$.

Definition 2.5. We say that a $J_{b}$-holomorphic map $f$ is $J_{B}$-parametric regular if $D \Phi(b, f)$ is onto.

Observation. The linearized operator is well defined if there is no pair $(b, f)$ with $f$ a $J_{b}$-holomorphic and $b \in \partial B$. This is precisely the condition we imposed on $\left(J_{B}, \partial J_{B}\right)$ to give a relative cycle in $\left(\mathscr{A}_{I}, \mathscr{A}_{I, D}^{c}\right)$.

Definition 2.6. Consider $\left(J_{B}, \omega_{B}\right)$ as above. We say that $\left(J_{B}, \partial J_{B}\right)$ is a $D$ parametric regular family of almost complex structures if any $J_{b}$-holomorphic map in class $D$ is parametric regular. We denote by $J_{\text {preg }}(D)$ the set of all D-parametric regular families $\left(J_{B}, \partial J_{B}\right) \subset\left(\mathscr{A}_{I}, \mathscr{A}_{I, D}^{c}\right)$.

To apply the implicit function theorem and the Sard-Smale theorem, we must work on Banach manifolds and hence complete all spaces under suitable Sobolev norms. For example, one should work on spaces consisting of almost complex structures of class $C^{l}$, on $\mathscr{X}^{k, p}$, with $k p>2$, the space of maps whose $k$-th derivatives are of class $L^{p}$. Also, we should work on

$$
\mathscr{E}_{f}^{p}=L^{p}\left(\Lambda^{0,1} \otimes_{J} f^{*} T M\right)
$$

rather that with $\Omega_{J}^{0,1}\left(\Sigma, f^{*} T M\right)$. There are standard arguments [McDuff and Salamon 1994] to show that one can transfer the following arguments from spaces of $C^{l}$ objects (which are Banach manifolds) to spaces of $C^{\infty}$ objects (which are Fréchet manifolds). For simplicity we will drop the superscripts $l, k, p$ unless specifying them is relevant.

Theorem 2.7. If $J_{B} \in J_{\text {preg }}(D)$, the moduli space $\widetilde{\mathcal{M}}_{0,0}^{*}\left(M, D,\left(J_{B}, \partial J_{B}\right)\right)$ is a smooth open manifold of dimension $2 n+2 c_{1}(D)+\operatorname{dim} B$, with a natural orientation.

Moreover, if one considers $\widetilde{M}_{0,0}^{*}\left(M, D,\left(J_{B}, \partial J_{B}\right)\right) \times\left(S^{2}\right)^{k}$ and takes away all the diagonals of the type $\widetilde{M}_{0,0}^{*}\left(M, D,\left(J_{B}, \partial J_{B}\right)\right) \times \operatorname{diag}_{i, j}$, one obtains precisely $\widetilde{M}_{0, k}^{*}\left(M, D,\left(J_{B}, \partial J_{B}\right)\right)$. This will therefore be a manifold of dimension $2 n+$ $2 c_{1}(D)+\operatorname{dim} B+2 k$.

Let $\widetilde{M}_{0,0}^{*}\left(M, D, \mathscr{A}_{I}\right)$ be the universal moduli space consisting of pairs $(f, J)$, where $J \in \mathscr{A}_{I}$ and $f$ is $J$-holomorphic. It will be relevant for the results we have in mind to point out the following characterization of parametric regularity. 
Proposition 2.8. Consider the diagram

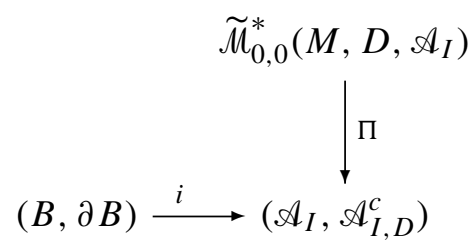

Then $J_{B} \in J_{\text {preg }}(A)$ if and only if $i \pitchfork \Pi$.

Proof. For simplicity we will write $D_{f, b}=D \Phi(b, f)_{\mid C^{\infty}\left(f^{*}(T M)\right)}$. By (9), the surjectivity of $D \Phi(b, f)$ is then equivalent to the surjectivity of the linear operator

$$
D \phi_{\mid T_{b} B}: T_{b} B \rightarrow \operatorname{coker} D_{b, f} .
$$

We will set $i(b)=J$. The tangent space $T_{J} \mathscr{A}_{I}$ to $\mathscr{A}_{I}$ consists of all sections $Y$ of the bundle $\operatorname{End}(T M, J)$ whose fiber at $p \in M$ is the space of linear maps $Y: T_{p} M \rightarrow T_{p} M$ such that $Y J+J Y=0$; we will consider the map

$$
R: T_{J} \mathscr{A}_{I} \rightarrow \Omega_{J}^{0,1}\left(\Sigma, f^{*} T M\right)
$$

given by $R(Y)=\frac{1}{2} Y \circ d f \circ j$. The map

$$
d \Pi: T_{f, J} \widetilde{M}_{0,0}^{*}\left(M, D, \mathscr{A}_{I}\right) \rightarrow T_{J} \mathscr{A}_{I}
$$

is given by $d \Pi(\xi, Y)=Y$, where the pair $(\xi, Y)$ is in $T_{f, J} \widetilde{\mathcal{M}}_{0,0}^{*}\left(M, D, \mathscr{A}_{I}\right)$ if and only if

$$
D_{f, b}(\xi)+R(Y)=0 .
$$

From this one can see that $\operatorname{im} D_{f, b}=R(\operatorname{im} d \Pi)$. Since $D_{b, f}$ is elliptic and ker $R \subset$ $\operatorname{im} d \Pi$, it follows that coker $d \Pi$ has finite dimension. If we consider the map $\mathscr{F}: \mathscr{X} \times \mathscr{A}_{I} \rightarrow \mathscr{E}$, given by $\mathscr{F}(f, J)=\bar{\partial}_{J}(f)$ then (see [McDuff and Salamon 1994]) the linearization at a zero $(f, J)$ with $f$ simple is onto. That is

$$
D \mathscr{F}(f, J)(\xi, Y)=D_{f} \xi+R(Y)
$$

is onto. This implies that coker $D_{f}$ is covered by $R$. We can show that there is an induced map

$$
\tilde{R}: \operatorname{coker} d \Pi \rightarrow \operatorname{coker} D_{b, f}
$$

which is isomorphism. We have $D \Phi_{\mid T_{b} B}(Y)=R \circ d i$, so

$$
i \pitchfork \Pi \Longleftrightarrow d i \rightarrow \operatorname{coker} d \Pi \text { onto } \Longleftrightarrow \tilde{R} \circ d i \rightarrow \operatorname{coker} D_{b, f} \text { onto. }
$$

The proposition follows. 
We call attention to a few key points. Parametric regularity is a generalization of the usual regularity. Indeed, if we consider $J_{b}=J$ to be constant for $b$ in a neighborhood around $b_{0}$, the regularity of an almost complex structure $J$ simply says, following the diagram above, that $d \Pi$ is surjective. If we now regard $J$ within an arbitrary family $J_{B}$, this no longer needs to be the case. It will then suffice that the cokernel of $d \Pi$ is covered by the variation of $J$ in the direction of $B$.

In fact, when we count rational maps, the criterion of parametric regularity described below reduces the problem to the usual regularity in some suitable ambient space.

More precisely, note that the regularity of a holomorphic map is a local statement within $B$ and it only concerns the almost complex structure data. Therefore, for each $b \in \stackrel{\circ}{B}$, we can restrict our attention to a neighborhood of $b$, and without loss of generality the following discussion can be made for smoothly trivial fibrations. We say that a family $\left(J_{B}, \omega_{B}\right)$ descends from a fibration $M \rightarrow \widetilde{M} \rightarrow B$ if there is a diagram

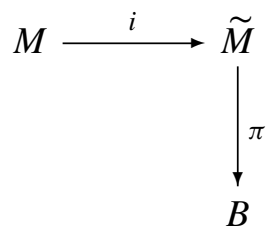

such that the almost complex structure $\tilde{J}$ on $\tilde{M}$ yields, by restriction to each fiber $M \times b$, the almost complex structure $J_{b}$ on $M$, and such that the closed two-form $\widetilde{\omega}$ on $\tilde{M}$ also gives, by restriction to each fiber, the symplectic form $\omega_{b}$, which tames $J_{b}$. Here we have chosen a trivialization of the fibration such that $\widetilde{M}=B \times M$ smoothly and $\pi$ is just the projection on the first factor. In the following theorem we consider the family of parameters $B$ to be a subset of $\mathbb{C}^{m}$ and we denote by $z$ the parameter.

Theorem 2.9. Let $\left(J_{z}, \omega_{z}\right)_{z \in B \subset \mathbb{C}^{m}}$ be a family on $M$ descending from the symplectic fibration $(\tilde{M}, \tilde{J}, \widetilde{\omega})$. Suppose that $f: \Sigma \rightarrow M$ is a $J_{0}$-holomorphic map and consider the composite map

$$
\tilde{f}=i \circ f, \quad \tilde{f}: \Sigma \rightarrow M \times 0 \subset \tilde{M},
$$

which is $\tilde{J}$-holomorphic. If $\tilde{f}$ is regular, $f$ is $\left(J_{z}\right)$-parametric regular. If $\Sigma=S^{2}$, the reverse statement also holds.

For the proof of the Theorem see the Appendix.

There exists a large subset of parametric regular families of almost complex structures inside $\left(\mathscr{A}_{I}, \mathscr{A}_{I, D}^{c}\right)$. This is because one can employ the Sard-Smale theorem [Smale 1965] and show that any map $i:(B, \partial B) \rightarrow\left(\mathscr{A}_{I}, \mathscr{A}_{I, D}^{c}\right)$ in Proposition 2.8 can be perturbed to an $i^{\prime}$ such that $i^{\prime} \pitchfork \Pi$. 
Definition 2.10. We will say that $\left(J_{B}, \partial J_{B}\right)$ satisfies hypothesis $H_{1}$ if it is a $D$ parametric regular family of almost complex structures.

Compactness. Even in those situations when (P1) is easily achieved using SardSmale, (P2) is seldom true. However, (P2) is true when $k$ is either 0 or 1 , and the class $D$ is $J_{b}$ indecomposable for any $b \in B$. This means that no $J_{b}$-holomorphic map in class $D$ can decompose into a connected union of $J_{b}$-holomorphic spheres $C=C^{1} \cup C^{2} \cup \cdots \cup C^{N}$ such that each $C^{i}$ represents the class $D_{i}$ and $D=$ $D_{1}+\cdots+D_{N}$. Then as a consequence of Gromov's compactness theorem it follows that $\mathcal{M}_{0, k}^{*}:=M_{0, k}^{*}\left(M, D,\left(J_{B}, \partial J_{B}\right)\right)$ is compact and hence in this situation the image of $e v: M_{0, k}^{*}\left(M, D,\left(J_{B}, \partial J_{B}\right)\right) \rightarrow M^{k}$ is a cycle.

Definition 2.11. We will say that the hypothesis $H_{2}$ is satisfied by $\left(J_{B}, \partial J_{B}\right)$ and $D$ if the class $D$ is $J_{b}$ indecomposable for every $b \in B$.

Note that if $D$ is $J_{b}$-indecomposable and $k \geq 2$ then in order to compactify the image of the evaluation map one only needs to add the limits of sequences of $J$ holomorphic maps for which two distinct marked points converge to each other. Hence $e v\left(M_{0, k}^{*}\right)$ will have boundary of codimension 2 or more and hence it will carry a fundamental class.

\section{Resolutions of singularities and relative PGW}

Quotient singularities. We now give an overview of work of Kronheimer [1998] and Abreu and McDuff [2000] on how to construct special families of almost complex structures arising from the study of the total spaces of deformations for some quotient singularities. At the end of the section we will explain how these families serve our purpose of counting nontrivial PGWs. The local picture is as follows [Kronheimer 1998]:

We consider the particular type of Hirzebruch-Jung singularity $Y_{0}=\mathbb{C}^{2} / C_{2 \ell}$, given by the diagonal action by scalars of $C_{2 \ell}$ on $\mathbb{C}^{2}$, where $C_{2 \ell}$ is the cyclic group of order $2 \ell$. This admits a resolution $\sigma_{0}: \widetilde{Y}_{0} \rightarrow Y_{0}$, where $\widetilde{Y}_{0}$ is the total space of the line bundle of degree $-2 \ell$ over $\mathbb{C} P^{1}$. The exceptional curve of the resolution, we will call it $\mathrm{E}$, is a curve of self-intersection $-2 \ell$ and is the zero section of $\tilde{Y}_{0}$. This resolution admits a $(2 \ell-1)$-complex-dimensional parameter family of deformations $\tilde{Y}_{t}, t \in \mathbb{C}^{2 \ell-1}$. With the exception of the case $\ell=2$ the total space $\widetilde{Y}=\bigcup \widetilde{Y}_{t}$ of the family of deformations is the total space of the vector bundle $\mathcal{O}(-1)^{2 \ell}$. More precisely, we consider the exact sequence of bundles

$$
\mathrm{O}(-2 \ell) \longrightarrow \mathrm{O}(-1)^{2 \ell} \stackrel{r}{\longrightarrow} \mathrm{O}^{2 \ell-1},
$$

where $r$ is given by evaluating at $2 \ell-1$ generic sections of the dual, $\widetilde{Y}^{*}=O(1)^{2 \ell}$ of $\tilde{Y}$. Since holomorphically $0^{2 \ell-1}$ is trivial, we can project it to its fiber $\mathbb{C}^{2 \ell-1}$ 
and hence obtain a submersion $\tilde{q}: \mathcal{O}(-1)^{2 \ell} \rightarrow \mathbb{C}^{2 \ell-1}$ with $\widetilde{Y}_{t}=\tilde{q}^{-1}(t)$. Also it can be seen that $\tilde{Y}$ is diffeomorphic with $\widetilde{Y}_{0} \times \mathbb{C}^{2 \ell-1}$ and a choice of trivialization provides a fiberwise diffeomorphism

$$
\theta: \widetilde{Y} \stackrel{C^{\infty}}{=} \tilde{Y}_{0} \times \mathbb{C}^{2 \ell-1},
$$

where $\widetilde{Y}_{0}$ is the total space of the bundle $O(-2 \ell)$. Now consider a $4 \ell$-dimensional basis of sections in the dual $\widetilde{Y}^{*}$. Here the space of holomorphic sections is given by $\bigoplus_{i=1}^{2 \ell} H^{0}\left(C P^{1}, \mathcal{O}(1)\right) \tilde{=}\left(\mathbb{C}^{2}\right)^{2 \ell}$. Denote by $Y$ the subspace of $\left(\mathbb{C}^{2}\right)^{2 \ell}$ consisting of $2 \ell$-tuples of vectors in $\mathbb{C}^{2}$ spanning either zero or a line. By evaluating all the $4 \ell$ section we obtain a map

$$
\sigma: \widetilde{Y} \longrightarrow Y \subset \mathbb{C}^{4 \ell}
$$

that contracts $E$ to a point $\gamma_{0}=\sigma(E)$. Moreover, $\gamma_{0}$ is the only singular point of $Y$ and the morphism is one-to-one outside $E$. Define a map $q: Y \rightarrow \mathbb{C}^{2 \ell-1}$ by evaluating at the original $2 \ell-1$ generic sections. The diagram

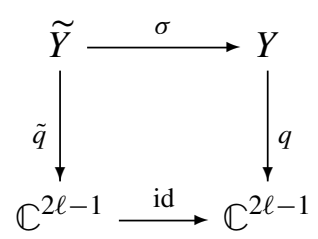

commutes. We can obtain a two-form $\tau$ on $Y$ by pulling back a Kähler form from $\mathbb{C}^{4 \ell}$. Via $\sigma^{*}$ this can be seen as a two-form on $\widetilde{Y}$ that restricts to a Kähler form $\tau_{t}$ on each fiber $\widetilde{Y}_{t}$ if $t \neq 0$ but degenerates along $E$ when $t=0$. If we further push forward through $\theta$, these forms can be seen as a family of forms on $\widetilde{Y}_{0}$.

As in [Abreu and McDuff 2000], we can choose an appropriate compactification of the local picture as follows:

Let $B^{4 \ell-2}$ be the unit ball in $\mathbb{C}^{2 \ell-1}$. We have a family $\left(\bar{Y}_{t}, J_{t}^{\ell}, \tau_{t}\right)_{t \in B^{4 \ell-2}}$, where each $\left(\bar{Y}_{t}, J_{t}^{\ell}, \tau_{t}^{\ell}\right), t \neq 0$ is a Kähler manifold diffeomorphic with $S^{2} \times S^{2}$, and, $\left(\bar{Y}_{0}, J_{0}^{\ell}\right)$ is a complex manifold, also diffeomorphic with $S^{2} \times S^{2}$ and $\tau_{0}$ degenerates along $E$ which represents the homology class $A-\ell F$. We take $A=\left[S_{\text {base }}^{2}\right]$ and $F=\left[S_{\text {fiber }}^{2}\right]$. The total space of the family has the following properties:

- The space $\bar{Y}=\cup_{t \in B^{4 \ell-2}} \bar{Y}_{t}$ is smoothly diffeomorphic to $S^{2} \times S^{2} \times B^{4 \ell-2}$. Moreover $\bar{Y}$ is a complex manifold with a complex structure $\tilde{J}^{\ell}$ which restricts to each fiber $\bar{Y}_{t}$ to the complex structure $J_{t}^{\ell}$. Also, $\bar{Y}$ has a closed $(1,1)$ form $\tau$ which is satisfies all the properties of a Kähler form outside the zero fiber and restricts at each fiber to the forms $\tau_{t}$.

- The restriction of $\tau$ to $\bar{Y}_{0}$ degenerates along the curve $E$ representing the class $A-\ell F$. 
Since the forms $\tau_{t}$ are obtained by restricting the closed form $\tau$ to fibers, it is clear that they are all in the same cohomology class. From $\left(\tau_{0}\right)_{\mid E}=0$ we have $\left[\tau_{0}\right](A-\ell F)=0$, and hence $\left[\tau_{t}^{\ell}\right]=\left[\omega_{\ell}\right]$ for all $t \in B^{4 \ell-2}$, where, as in the introduction, $\omega_{\ell}=\sigma_{F} \oplus \ell \sigma_{B}$ is a symplectic form on $S^{2} \times S^{2}$.

From (a) we see that there is a holomorphic projection $\pi: \bar{Y} \rightarrow S^{2} \times B^{4 \ell-2}$. This is because every $\bar{Y}_{t}$ is a ruled surface therefore it fibers over $S^{2}$. If we denote by $\alpha$ the area form on $S^{2}$ we can construct a two-form

$$
\tau^{\lambda}=\tau+(\lambda-\ell) \pi^{*}(\alpha)
$$

For $\lambda>\ell$ these forms are Kähler forms, and restricted to each $\bar{Y}_{t}$ they yield symplectic forms in the class $\left[\omega_{\lambda}\right]$. This proves that any $J_{t}^{\ell}$ (including $J_{0}^{\ell}$ ) is tamed by a form isotopic to $\omega_{\lambda}$, as long as $\lambda>\ell$. We now follow a similar procedure to construct a family of symplectic forms $\omega_{t}$, for $t \in B^{4 \ell-2}$, such that each $\omega_{t}$ tames $J_{t}^{\ell}$. We next change the forms $\tau_{t}$ by perturbing with a a positive factor of $\pi^{*}(\alpha)$ only around $t=0$ and smooth with a cutoff function. With this procedure we obtain symplectic forms $\omega_{t}$ with variable cohomology classes.

In conclusion, we have pairs

$$
\left(S^{2} \times S^{2}, J_{t}^{\ell}, \omega_{t}\right)_{t \in B^{4 \ell-2},}
$$

where $\omega_{t}$ is a symplectic structure on $S^{2} \times S^{2}$ that tames $J_{t}^{\ell}$. Moreover $\left[\omega_{t}\right]_{t \in S^{4 \ell-3}}=$ $\left[\omega_{\ell}\right]$. This gives a family of almost complex structures (which we denote $B_{\ell}$, by abuse of notation) such that $\left(B_{\ell}, \partial B_{\ell}\right) \in\left(\mathscr{A}_{[\ell, \ell+\epsilon]}, A_{\ell}\right)$ for any $\epsilon>0$. More importantly, for cohomological reasons, only $J_{0}^{\ell}$ admits almost holomorphic stable curves in the class $A-\ell F$.

We then obtain a family of almost complex structures on $\left(S^{2} \times S^{2} \times X\right)$ by taking $\left(J_{t}^{\ell} \times J_{\mathrm{arb}}\right)$, and by abuse of notation, we call this family also $B_{\ell}$. Thus we have just produced on $\left(S^{2} \times S^{2} \times X\right)$ pairs $\left(B_{\ell}, \partial B_{\ell}\right) \subset\left(A_{[\ell, \ell+\epsilon]}, A_{\ell}\right)$, with $\epsilon>0$, representing an element $\beta_{\ell}$ in $\pi_{*}\left(\mathscr{A}_{[\ell, \ell+\epsilon]}, A_{\ell}\right)$. Moreover each $B_{\ell}$ is contained in $A_{\ell+\epsilon}$ for any small $\epsilon>0$.

From the choice of the $J$ 's we know that the only almost complex structure admitting $A-\ell F$ almost complex stable curves is $J_{0} \times J_{\text {arb }}$.

The computation of $\boldsymbol{P} \boldsymbol{G W}$. Here we prove that $\left(H_{1}\right)$ and $\left(H_{2}\right)$ are satisfied for the family $\left(B_{\ell}, \partial B_{\ell}\right)$, and therefore the invariant is integer-valued and can be obtained by counting holomorphic maps intersecting generic cycles of appropriate dimension.

Claim 1. The family $\left(B_{\ell}, \partial B_{\ell}\right)$ satisfies $H_{1}$.

Proof. From the sequence (13) we see that the exceptional curve $E$, which is $\tilde{J}^{\ell}$ holomorphic, has normal bundle $\mathcal{O}(-1)^{2 \ell}$; therefore we can apply [McDuff and Salamon 1994, Lemma 3.5.1, p. 38] for the integrable almost complex structure $\tilde{J}$. 
If follows that $E$ is $\tilde{J}^{\ell}$-regular inside $\bar{Y}$. If we now consider $\bar{Y} \times X$ and $\tilde{J}^{\ell} \times J_{\text {arb }}$, the curve $E$ lies entirely inside $\bar{Y}$ and therefore the normal bundle inside $\bar{Y} \times X$ is $\mathcal{O}(-1)^{2 \ell} \times$ trivial, and therefore the curve is $\left(\tilde{J}^{\ell} \times J_{\text {arb }}\right)$-regular. This splitting and therefore regularity use the fact that the map $\mathrm{E}$ is of genus zero. Theorem 2.9 implies parametric regularity and therefore $\left(H_{1}\right)$ holds.

Claim 2. The family $\left(B_{\ell}, \partial B_{\ell}\right)$ satisfies $H_{2}$.

Proof. This is proved by inspection. Only $J_{0}^{\ell} \times J_{\text {arb }}$ admits $(A-\ell F)$-stable maps, and the only maps in this class are copies of the embedded map $E$ in any fiber $S^{2} \times S^{2} \times$ pt. Hence there are no decomposable $J_{b}$-holomorphic maps.

Remark. For other almost complex structures $J$ on $S^{2} \times S^{2} \times X$ one could have decomposable $J$-holomorphic maps in the class $A-\ell F$. For an example, consider $M=S^{2} \times S^{2} \times \mathbb{C} P^{n}$ and $\omega=\omega_{1+\epsilon} \oplus \omega_{\mathrm{arb}}$. If $H$ denotes the hyperplane class in $\mathbb{C} P^{n}$, we can take $\omega_{\text {arb }}$ such that $\omega(A-F-H)>0$ and get a symplectic embedding of $S^{2}$ into $M$, in the class $A-F-H$. We can choose an $\omega$-tamed almost complex structure $\tilde{J}$ on $M$ that fibers over the base $S^{2} \times S^{2}$ and such that the class $H$ has a $\tilde{J}$-holomorphic representative. Then the class $A-F$ is $\tilde{J}$-decomposable, where the decomposition is given by a $C$ with $C=C_{1} \cup C_{2}$ with $\left[C_{1}\right]=A-F-H$ and $\left[C_{2}\right]=H$.

We conclude that the invariants

$$
\mathrm{PGW}_{A-\ell F, 0, k}^{S^{2} \times S^{2} \times X,\left(B_{\ell}, \partial B_{\ell}\right)}: \bigoplus_{i=1}^{k} H^{a_{i}}\left(S^{2} \times S^{2} \times X, \mathbb{Q}\right)^{k} \rightarrow \mathbb{Z}
$$

are integer-valued. We have two situations. First, if $X=\mathrm{pt}$, the moduli space of unparametrized curves has dimension 0 , so we would count isolated curves. This follows immediately from the equality $c_{1}(A-\ell F)=-4 \ell+2$ (adjunction formula), so that

$$
\begin{aligned}
\operatorname{dim} M_{0,0}^{*}\left(S^{2} \times S^{2}, A-\ell F,\left(B_{\ell}, \partial B_{\ell}\right)\right) & =2 \times 2+2 c_{1}(A-\ell F)+\operatorname{dim} B^{\ell}-6 \\
& =4-4 \ell+4+4 \ell-2-6=0 .
\end{aligned}
$$

Moreover, the invariant $\mathrm{PGW}_{A-\ell F, 0,0}^{S^{2} \times S^{2} \times X,\left(B_{\ell}, \partial B_{\ell}\right)}([\mathrm{pt}])$ equals 1 because it counts $E$, the only $J_{b}$-map (where $b \in B^{\ell}$ ) in the class $A-\ell F$.

In the situation $\operatorname{dim} X=2 n>0$, we will count maps with one marked point. Then $c_{1}(A-\ell F)$ is the same, since the holomorphic maps in class $A-\ell F$ will be copies of the curve $E$ and hence will have the image entirely in the fibers $S^{2} \times S^{2} \times$ pt $\subset$ $S^{2} \times S^{2} \times X$. We therefore have

$\operatorname{dim} \mathcal{M}_{0,1}^{*}\left(S^{2} \times S^{2} \times X, A-\ell F,\left(B_{\ell}, \partial B_{\ell}\right)\right)=2 \times(2+n)+2 c_{1}(A-\ell F)+\operatorname{dim} B^{\ell}-6+2$

$$
=2 n+2 \text {. }
$$


We consider a cycle in the homology class $F$ lying in a fiber $S^{2} \times S^{2} \times \mathrm{pt}$ inside $S^{2} \times S^{2} \times X$. It easily follows that the only $J_{b_{\ell}}$-holomorphic map with one marked point that intersects this cycle transversely is a copy of the map $E$ inside the fiber $S^{2} \times S^{2} \times$ pt. We obtain

$$
\mathrm{PGW}_{A-\ell F, 0,1}^{S^{2} \times S^{2} \times X,\left(B_{\ell}, \partial B_{\ell}\right)}(P D([F]))= \pm 1,
$$

where the sign depends on the orientation of the parameter space $B_{\ell}$. Applying Theorem 2.2 we conclude that the morphism $\Theta$ in both situations is nontrivial and therefore there is a nonzero element

$$
\beta_{\ell} \in \pi_{4 \ell-2}\left(\left(\mathscr{A}_{[\ell, \ell+\epsilon]}, A_{\ell}\right)\right) \quad \text { for all } \epsilon>0
$$

represented by the cycle $\left(B_{\ell}, \partial B_{\ell}\right) \subset\left(\mathscr{A}_{\ell+\epsilon}, \mathscr{A}_{\ell+\epsilon, D}^{c}\right)$.

\section{Almost complex structures and symplectomorphism groups}

Almost complex structures and symplectomorphisms; deformations along compact subsets. We now give a quick overview of what can be said about the behavior of spaces of almost complex structures and about the symplectomorphism groups as the symplectic form varies along the line $L$.

If $L$ happens to be a ray $\lambda \omega, \lambda>0$, then $G_{\lambda}$ is independent of $\lambda$. Thus we may as well assume $L$ is not a ray.

If $M=S^{2} \times S^{2}$, much is known about the structure of $\mathscr{A}_{\lambda}$; see [McDuff 2000]. For example, one can establish that there is a direct inclusion $\mathscr{A}_{\lambda} \subset \mathscr{A}_{\lambda^{\prime}}$, for $\lambda<\lambda^{\prime}$. Moreover, the homotopy type of the spaces $\mathscr{A}_{\lambda}$ changes only as $\lambda$ strictly passes an integer $\ell$.

None of this is known to hold when $M$ is an arbitrary symplectic manifold. Nevertheless, as a consequence of the fact that taming is an open condition, we are able to establish the following lemma, which we use in the proof of Theorem 2.9.

Lemma 4.1. (a) Let $K^{\prime}$ to be an arbitrary compact subset of $\mathscr{A}_{\lambda}$. There is an $\epsilon_{K^{\prime}}>0$ such that $K^{\prime}$ is contained in $\mathscr{A}_{\lambda+\epsilon}$, for $|\epsilon|<\epsilon_{K^{\prime}}$.

(b) Consider $K$ an arbitrary compact set in $G_{\lambda}$. For $\varphi_{G}$ as in (4), there is an $\epsilon_{K}>0$ and a map $h:\left[-\epsilon_{K}, \epsilon_{K}\right] \times K \rightarrow \mathscr{G}_{\mid L}$ such that the diagram

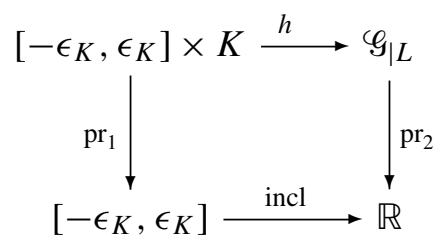

commutes. 
For any two such maps $h$ and $h^{\prime}$ coinciding on $0 \times K$, there exists, for $\epsilon^{\prime}$ small enough, a homotopy $H:[0,1] \times\left[-\epsilon^{\prime}, \epsilon^{\prime}\right] \times K \rightarrow \mathscr{G}_{\mid L}$ between them that satisfies

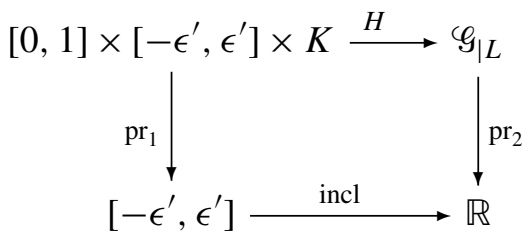

Proof. Part (i) is an immediate consequence of the openness of the taming condition.

For the proof of (ii), let's first notice that, since the symplectic condition is an open condition, there is a convex open neighborhood $U$ of $\omega_{\lambda}$ inside the space of 2-forms such that any closed $\omega^{\prime}$ in $U$ is still symplectic.

Moreover since $K$ is compact there is an $\epsilon(K)>0$ such that, for any $g_{k} \in K$,

$$
g_{k}^{*} \omega_{\lambda+\epsilon} \in U \text { for all } 0 \leq \epsilon<\epsilon(K) .
$$

This is true because we can produce such an $\epsilon$ for an open set around each element $g \in K$ and hence find a global $\epsilon(K)$ by following a standard compactness argument.

We will construct the elements $h(\epsilon, k)$ as follows. For $t \in[0,1]$ the forms

$$
\omega_{k, \lambda+\epsilon}^{t}:=\operatorname{tg}_{k}^{*} \omega_{\lambda+\epsilon}+(1-t) \omega_{\lambda+\epsilon}
$$

are symplectic, since both $g_{k}^{*} \omega_{\lambda+\epsilon}$ and $\omega_{\lambda+\epsilon}$ are inside the convex set $U$. Moreover, since $K \subset G_{\lambda} \subset \operatorname{Diff}_{0} M$, any $g_{k}$ is smoothly isotopic to the identity and hence $\left[g_{k}^{*} \omega_{\lambda+\epsilon}\right]=\left[\omega_{\lambda+\epsilon}\right]$. Therefore the forms $\omega_{k, \lambda+\epsilon}^{t}$ are cohomologous as we vary $t$. We now apply Moser's argument for the one-parameter family of symplectic forms $\omega_{k, \lambda+\epsilon}^{t}$ and obtain a family of diffeomorphisms $\xi_{k, \lambda+\epsilon, t}$ having the property that $\xi_{k, \lambda+\epsilon, t}^{*} \omega_{k, \lambda+\epsilon}^{t}=\omega_{\lambda+\epsilon}$. We next define $h(\epsilon, k):=g_{k} \circ \xi_{k, \lambda+\epsilon, 1}$. Then $h$ has the required properties.

For an arbitrary $h:[-\epsilon, \epsilon] \times K$ satisfying (17) we take the homotopy

$$
F:[0,1] \times[-\epsilon, \epsilon] \times K \rightarrow \mathbb{R} \times \operatorname{Diff}_{0} M
$$

given by $F(t, \epsilon, k):=(\epsilon, h(t \epsilon, k))$.

This gives a homotopy between $h$ and $h_{0}:[-\epsilon, \epsilon] \times K \rightarrow \mathbb{R} \times \operatorname{Diff}_{0} M$, where $h_{0}\left(\epsilon^{\prime}, k\right)=h(0, k)$. We similarly obtain a homotopy $F^{\prime}$ between $h^{\prime}$ and $h_{0}$, where $h^{\prime}$ also satisfies (17). By concatenating one homotopy with the opposite of the other we obtain a homotopy between $h$ and $h^{\prime}$, which we call $G:[0,1] \times\left[-\epsilon_{1}, \epsilon_{1}\right] \times K \rightarrow$ $\mathbb{R} \times \operatorname{Diff}_{0} M$. We set $g_{s, \epsilon, k}:=G(s, \epsilon, k)$ and follow the same procedure as before: we restrict to a short interval $\left[-\epsilon^{\prime}, \epsilon^{\prime}\right]$ such that, if we define

$$
\omega_{s, k, \lambda+\epsilon}^{t}:=\operatorname{tg}_{s, \epsilon, k}^{*} \omega_{\lambda+\epsilon}+(1-t) \omega_{\lambda+\epsilon},
$$


these maps are symplectic for all $0 \leq|\epsilon|<\epsilon^{\prime}$ and $t, s \in[0,1]$. This is possible because $\omega_{s, k, \lambda}^{t}=\omega_{\lambda}$. Again, the diffeomorphisms $g_{s, \epsilon, k}$ are smoothly isotopic to the identity and, as above, we can apply Moser's argument to the isotopic forms $\omega_{s, k, \lambda+\epsilon}^{t}$, to obtain diffeomorphisms $\xi_{s, k, \lambda+\epsilon, t}$ such that $\xi_{s, k, \lambda+\epsilon, t}^{*} \omega_{s, k, \lambda+\epsilon,}^{t}=\omega_{\lambda+\epsilon}$. If we define $H(s, \epsilon, k):=g_{s, \epsilon, k} \circ \xi_{s, k, \lambda+\epsilon, 1}$, the map $H$ has the required properties.

Definition 4.2. Let $\rho: B \rightarrow G_{\lambda}$ be a cycle in $G_{\lambda}$. An extension $\rho^{\epsilon}$ of $\rho$ is a smooth family of cycles $\rho^{\epsilon}: B \rightarrow G_{\lambda+\epsilon}$ defined for $|\epsilon| \leq \epsilon_{0}$ such that $\rho^{0}=\rho$ and satisfying (18). Using Lemma 4.1(i) we see that every cycle $\rho$ has an extension.

Observation. Consider two extensions $\rho_{1}^{\epsilon}$, where $0 \leq|\epsilon|<\epsilon_{1}$, and $\rho_{2}^{\epsilon}$, where $0 \leq|\epsilon|<\epsilon_{2}$. By (18) there is an $\epsilon^{\prime}>0$ and a homotopy between $\rho_{1}^{\epsilon}$ and $\rho_{2}^{\epsilon}$ defined for all $0 \leq \epsilon \leq \epsilon^{\prime}$. Hence any extension provides well defined elements in $\pi_{*}\left(G_{\lambda+\epsilon}\right)$ for small values of $\epsilon$. Therefore each $[\rho] \in \pi_{*}\left(G_{\lambda}\right)$ has an extension $\left[\rho^{\epsilon}\right] \in \pi_{*}\left(G_{\lambda+\epsilon}\right)$ whose germ at $\epsilon=0$ is independent of the choices of $\rho$.

Definition 4.3. We say that a smooth family of elements $\left[\rho^{\epsilon}\right] \in \pi_{*}\left(G_{\lambda+\epsilon}\right)$, with $0<\epsilon<\epsilon_{\rho}$, is new if it is not the extension for $\epsilon>0$ of any element $[\rho] \in \pi_{*}\left(G_{\lambda}\right)$.

In the next section we will use the same letter $\rho$ to refer both to cycles as well as to the homotopy class they represent.

Relation between almost complex structures and symplectomorphism groups; proof of Theorem 1.3. We consider the long exact sequence of relative homotopy groups of the pair $\left(\mathscr{A}_{\ell^{+}}, \mathscr{A}_{\ell}\right)$ :

$\cdots \longrightarrow \pi_{k}\left(\mathscr{A}_{\ell^{+}}\right) \longrightarrow \pi_{k}\left(\mathscr{A}_{\ell^{+}}, \mathscr{A}_{\ell}\right) \longrightarrow \pi_{k-1}\left(\mathscr{A}_{\ell}\right) \longrightarrow \pi_{k-1}\left(\mathscr{A}_{\ell^{+}}\right) \longrightarrow \cdots$

Since by construction $\beta_{\ell} \in \pi_{k}\left(\mathscr{A}_{\ell^{+}}, \mathscr{A}_{\ell}\right)$ is nontrivial, one of the two following cases can happen:

1. $\beta_{\ell} \mapsto \gamma_{\ell} \neq 0 \in \pi_{k-1}\left(\mathscr{A}_{\ell}\right)$.

2. $\beta_{\ell} \mapsto 0 \in \pi_{k-1}\left(\mathscr{A}_{\ell}\right)$. In this situation, there is a nonzero element $\alpha_{\ell} \in \pi_{k}\left(\mathscr{A}_{\ell^{+}}\right)$ that maps to $\beta_{\ell}$.

We analyze each case in turn:

Case 1. Consider the fibration (2), which yields $G_{\ell} \longrightarrow \operatorname{Diff}_{0} M \longrightarrow \mathscr{A}_{\ell}$, and then the long exact sequence in homotopy,

$\begin{aligned} \cdots \longrightarrow \pi_{k-1}\left(G_{\ell}\right) & \longrightarrow \pi_{k-1}\left(\operatorname{Diff}_{0} M\right) \longrightarrow \\ & \longrightarrow \pi_{k-1}\left(\mathscr{A}_{\ell}\right) \longrightarrow \pi_{k-2}\left(G_{\ell}\right) \longrightarrow \pi_{k-2}\left(\operatorname{Diff}_{0} M\right) \longrightarrow \cdots\end{aligned}$

Again, there are two possibilities: 
(i) $\gamma_{\ell} \rightarrow \theta_{\ell} \neq 0 \in \pi_{k-2}\left(G_{\ell}\right)$. In this situation, we have a nontrivial element $\theta_{\ell} \in \pi_{k-2}\left(G_{\ell}\right)$, such that $\theta_{\ell} \mapsto 0 \in \pi_{k-2}\left(\operatorname{Diff}_{0} M\right)$. Then we are in case (A) of Theorem 1.3.

This element is fragile. For assume it isn't; then $\theta_{\ell}$ can be extended by $\theta_{\ell+\epsilon}$, which yields nontrivial classes in $\pi_{k-2}\left(G_{\ell+\epsilon}\right)$. Then $\theta_{\ell+\epsilon} \mapsto 0 \in \pi_{k-2}\left(\operatorname{Diff}_{0} M\right)$ as well. Therefore $\theta_{\ell+\epsilon}$ appears as a boundary of an element $\gamma_{\ell+\epsilon} \in \pi_{k-1}\left(\mathscr{A}_{\ell+\epsilon}\right)$, which is homotopic to $\gamma_{\ell}$. But by construction and Lemma 4.1, we know that $\gamma_{\ell}$ is a contractible cycle inside $\mathscr{A}_{\ell+\epsilon}$. This contradicts the existence of $\gamma_{\ell+\epsilon}$.

(ii) $\gamma_{\ell} \mapsto 0 \in \pi_{k-2}\left(G_{\ell}\right)$. Then $\gamma_{\ell}$ is in the image of the morphism $\pi_{k-1}\left(\operatorname{Diff}_{0} M\right) \rightarrow$ $\pi_{k-1}\left(\mathscr{A}_{\ell}\right)$, so there is an element $\gamma_{\ell}^{\prime} \in \pi_{k-1}\left(\operatorname{Diff}_{0} M\right)$ such that $0 \neq \gamma_{\ell}^{\prime} \mapsto \gamma_{\ell}$.

In this situation, we can choose a cycle $S \subset \mathscr{A}_{\ell}$ representing $\gamma_{\ell} \in \pi_{k-1}\left(\mathscr{A}_{\ell}\right)$, and, using Lemma 4.1, there is an $\epsilon_{S}>0$ such that $S \subset \mathscr{A}_{\ell+\epsilon}$ for any $\epsilon \in\left(0, \epsilon_{S}\right)$. Now we claim that

$$
0=[S] \in \pi_{k-1}\left(\mathscr{A}_{\ell+\epsilon}\right) .
$$

For, by hypothesis, $S$ is the boundary of a cycle $B_{\ell}$ such that $B_{\ell} \subset A_{\ell+\epsilon}$ for all small $\epsilon>0$. Therefore we have a $k$-dimensional ball inside $A_{\ell+\epsilon}$ whose boundary is $S$, which proves the claim. We therefore have $\pi_{k-1}\left(\operatorname{Diff}_{0} M\right) \ni \gamma_{\ell}^{\prime} \mapsto[S]=0 \in$ $\pi_{k-1}\left(\mathscr{A}_{\ell+\epsilon}\right)$ on the top row of the commutative diagram

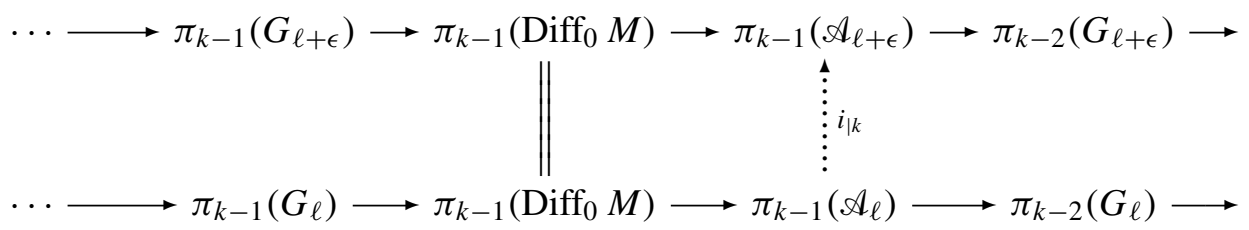

while on the bottom row the same $\gamma_{\ell}^{\prime}$ maps to $\gamma_{\ell} \in \pi_{k-1}\left(\mathscr{A}_{\ell+\epsilon}\right)$. By the exactness of the first row, $\gamma_{\ell}^{\prime}$ is in the image of the map $\pi_{k-1}\left(G_{\ell+\epsilon}\right) \rightarrow \pi_{k-1}\left(\operatorname{Diff}_{0} M\right)$, and therefore we are able to produce an element $0 \neq \eta_{\ell+\epsilon} \in \pi_{k-1}\left(G_{\ell+\epsilon}\right)$ such that $\eta_{\ell+\epsilon}$ persists in the topology of the group of diffeomorphisms. Thus we are in case (B).

The elements we obtain here are new. This follows easily by assuming the opposite. That is, if we consider that there is an element $0 \neq \eta_{\ell} \in \pi_{k-1}\left(G_{\ell}\right)$ whose germ is given by $\eta_{\ell+\epsilon}$, then the image of $\eta_{\ell}$ in $\operatorname{Diff}_{0} M$ has to be $\gamma_{\ell}^{\prime}$. But this contradicts the fact that $\gamma_{\ell}^{\prime} \mapsto \gamma_{\ell} \neq 0$.

Case 2. In this situation we have a nontrivial element $\alpha_{\ell} \in \pi_{k}\left(\mathscr{A}_{\ell^{+}}\right)$. Then we shall see that there is an $\epsilon$ such that for $0<\delta<\epsilon, \alpha_{\ell}$ has a representative $C$ inside $\mathscr{A}_{\ell+\delta}$, with $0 \neq[C] \in \pi_{k}\left(\mathscr{A}_{\ell+\delta}\right)$. The proof of this follows from the construction of $\alpha_{\ell}$. Namely, since $\beta_{\ell} \mapsto 0 \in \pi_{k-1}\left(\mathscr{A}_{\ell}\right)$, there exists a $k$-dimensional disk $D$ inside $A_{\ell}$ whose boundary is $\partial B_{\ell}$; by Lemma 4.1(i), this can be viewed inside $A_{\ell+\delta}$ for small $\delta$. We can now glue $B_{\ell}$ and $D$ along their boundary $\partial B_{\ell}$. In this manner we get a cycle $C \subset A_{\ell+\delta}$ representing the class $\alpha_{\ell}$. We can therefore consider again 
the sequence

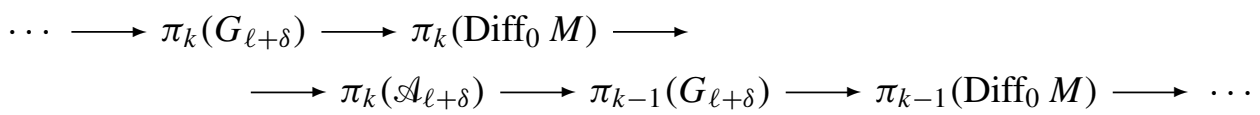

Next we claim that $[C]$ doesn't lift to a nontrivial element in $\pi_{k}\left(\operatorname{Diff}_{0} M\right)$. Indeed, there is a map

$$
\pi_{k}\left(\operatorname{Diff}_{0} M\right) \longrightarrow \pi_{k}\left(\mathscr{A}_{\lambda}\right)
$$

for any $\lambda$, and as $\lambda$ varies these maps vary homotopically in $\mathscr{A}_{I}$. If $C$ did lift, the map $\pi_{k}\left(\operatorname{Diff}_{0} M\right) \rightarrow \pi_{k}\left(\mathscr{A}_{\ell}\right)$ would produce a cycle $[B] \in \mathscr{A}_{\ell}$, which by means of Lemma 4.1 could be viewed inside all $\mathscr{A}_{\ell+\epsilon}$ for small $\epsilon$ and which moreover would be homotopic to $C$ inside $\mathscr{A}_{[\ell, \ell+\epsilon]}$. Therefore [C] would map to $0 \in \pi_{k}\left(\mathscr{A}_{\ell^{+}}, \mathscr{A}_{\ell}\right)$, contradicting its definition.

Since $[C]$ cannot be in the image of the map $\pi_{k}\left(\operatorname{Diff}_{0} M\right) \rightarrow \pi_{k}\left(\mathscr{A}_{\ell+\delta}\right)$, we know that $[C]$ must have nonzero image $[C] \mapsto \eta_{\ell+\delta} \neq 0$ in $\pi_{k-1}\left(G_{\ell+\delta}\right)$. Moreover, from the obvious properties of exact sequences again, $\eta_{\ell+\delta} \rightarrow 0$ through the natural inclusion map $\pi_{k-1}\left(G_{\ell+\delta}\right) \rightarrow \pi_{k-1}\left(\operatorname{Diff}_{0} M\right)$. That these elements are $n e w$ follows again by assuming the opposite. If they formed the germ of an element $\eta_{\ell}$ in $\pi_{k-1}\left(G_{\ell}\right)$, then $\eta_{\ell}$ would also be null-homotopic inside $\operatorname{Diff}_{0} M$, so it would come from a class $\left[C^{\prime}\right]$ in $\pi_{k}\left(\mathscr{A}_{\ell}\right)$. Moreover, $C^{\prime}$ would be homotopic with $C$ inside $\mathscr{A}_{[\ell, \ell+\delta]}$, therefore also in $\left(\mathscr{A}_{[\ell, \ell+\delta]}, \mathscr{A}_{\ell}\right)$, which is false given that $C$ has to yield a nontrivial element in $\pi_{k}\left(\mathscr{A}_{[\ell, \ell+\delta]}, \mathscr{A}_{\ell}\right)$. Thus we are in case (B) of the theorem.

With this, we have exhausted all the possible cases given by the nontrivial PGW, and the proof of Theorem 1.3 is complete.

Now to prove Corollary 1.4, consider the manifold $\left(S^{2} \times S^{2} \times X, \omega_{\lambda} \oplus \omega_{\text {arb }}\right)$. As seen in (16), the cycles $\left(B^{\ell}, \partial B^{\ell}\right)$ satisfy the definition (7), so by Proposition 2.4 they give persistent elements in $\pi_{4 \ell-2}\left(\mathscr{A}_{\ell^{+}}, \mathscr{A}_{\ell}\right)$. Therefore Theorem 1.3 applies and the corollary holds.

\section{Appendix: A proof of the criterion of parametric regularity, Theorem 2.9}

Let $T_{\left.\right|_{\pi^{-1}(0)}} \tilde{M}$ be the tangent space along the preimage of $0 \in \mathbb{C}^{m}$. Denote by $H$ the subbundle of $T_{\left.\right|_{\pi^{-1}(0)}} \tilde{M}$ which is $\widetilde{\omega}$-orthogonal to the fiber $\{0\} \times M$. We would like $H$ to coincide with the horizontal space of $T \tilde{M}$ with respect to the trivialization $\pi$ and to be $\tilde{J}$-invariant. This can be arranged by deforming the form $\widetilde{\omega}$ so that near the zero fiber $\{0\} \times M$ it is given by

$$
\widetilde{\omega}=\omega_{0}+\pi^{*}\left(\sigma_{\text {base }}\right),
$$


where $\sigma_{\text {base }}$ is a standard symplectic two-form on the holomorphic base $B$. Throughout this deformation process $\tilde{J}$ is still $\widetilde{\omega}$-tamed.

Let $g_{0}$ be a metric on $M_{0}$ and $\nabla$ the Levi-Civita connection on $M$ associated with it. Also let $\nabla^{s t}$ be the standard Levi-Civita connection on $\mathbb{C}^{m}$, and set $\widetilde{\nabla}=\nabla \times \nabla^{s t}$, the product connection on $\tilde{M} \simeq \mathbb{C}^{m} \times M$. The regularity of $\tilde{f}: \Sigma \rightarrow \tilde{M}$ is by definition equivalent to the surjectivity of $D_{\tilde{f}}$, the linearization of $\bar{\partial}$ :

$$
D_{\tilde{f}}: C^{\infty}\left(\tilde{f}^{*} T \tilde{M}\right) \longrightarrow \Omega_{\tilde{J}}^{0,1}\left(\Sigma, \tilde{f}^{*} T \tilde{M}\right) .
$$

Using the connection $\widetilde{\nabla}$ we will derive formulas for $D_{\tilde{f}}$ and express them in terms of the linearization $D \Phi$.

Since $\tilde{M} \simeq \mathbb{C}^{m} \times M$ and $\operatorname{im} \tilde{f} \subset\{0\} \times M$, we have the relations

$$
\tilde{f}^{*}(T \tilde{M})=\tilde{f}^{*}\left(T \tilde{M}_{\pi^{-1}(0)}\right)=\tilde{f}^{*}(H \oplus T M)=\operatorname{triv} \oplus f^{*}(T M),
$$

where by triv we denote the trivial $m$-dimensional complex bundle over $\Sigma$. This gives

$$
C^{\infty}\left(\tilde{f}^{*} T \tilde{M}\right) \simeq C^{\infty}(\text { triv }) \oplus C^{\infty}\left(f^{*} T M\right)
$$

Since each fiber is $\tilde{J}$-invariant and $H$ is $\tilde{J}$-invariant along $\pi^{-1}(0)$, we obtain

$$
\Omega_{\tilde{J}}^{0,1}\left(\Sigma, \tilde{f}^{*} T \tilde{M}\right) \simeq \Omega_{J}^{0,1}\left(\Sigma, f^{*} T M\right) \oplus \Omega_{\tilde{J}}^{0,1}(\Sigma, H) .
$$

From (20) and (21) we obtain

$$
D_{\tilde{f}}: C^{\infty}(\text { triv }) \oplus C^{\infty}\left(f^{*} T M\right) \longrightarrow \Omega_{J}^{0,1}\left(\Sigma, f^{*} T M\right) \oplus \Omega_{\tilde{J}}^{0,1}(\Sigma, H),
$$

and by considering the appropriate restrictions we obtain the operators

$$
\begin{array}{ll}
D_{1, \text { vert }}: C^{\infty}(\text { triv }) \rightarrow \Omega_{J}^{0,1}\left(\Sigma, f^{*} T M\right), & D_{2, \text { vert }}: C^{\infty}\left(f^{*} T M\right) \rightarrow \Omega_{J}^{0,1}\left(\Sigma, f^{*} T M\right), \\
D_{1, \text { hor }}: C^{\infty} \text { (triv) } \rightarrow \Omega_{\tilde{J}}^{0,1}(\Sigma, H), & D_{2, \text { hor }}: C^{\infty}\left(f^{*} T M\right) \rightarrow \Omega_{\tilde{J}}^{0,1}(\Sigma, H) .
\end{array}
$$

We sometimes write $D_{k}=\left(D_{k \text {,vert }}, D_{k \text {,hor }}\right)$, for $k=1,2$.

To compute the formulas for these operators we use a general method found in [Aebischer et al. 1994]: Consider $\xi \in C^{\infty}\left(\Sigma, \tilde{f}^{*} T \widetilde{M}\right)$ and $\widetilde{F}_{\xi}:[0,1] \times \Sigma \rightarrow \widetilde{M}$ given by $\widetilde{F}_{\xi}(t, x)=\exp \widetilde{\nabla}_{\tilde{f}(x)}(t \xi(x))$, for $\xi$ sufficiently small. Let $s: \Sigma \rightarrow T \Sigma$ be a section and let $\tilde{s}$ be its lift to $T([0,1] \times \Sigma)$. Denote by $\partial / \partial t$ the vector field in $T([0,1] \times \Sigma)$ corresponding to the parameter in $[0,1]$. Define $\tilde{f}_{t}(x):=\widetilde{F}_{\xi}(t, x)$. For any $x \in \Sigma$, define the path $\tilde{\gamma}_{x}^{\xi}:[0,1] \rightarrow \widetilde{M}$ given by $\tilde{\gamma}_{x}^{\xi}(t)=\widetilde{F}_{\xi}(t, x)$, the image under $\widetilde{F}_{\xi}$ of $[0,1] \times x$ in $\widetilde{M}$. By the definition of $\widetilde{F}_{\xi}, \tilde{\gamma}_{x}^{\xi}$ is a geodesic path in $\widetilde{M}$ relative to the connection $\widetilde{\nabla}$. Denote by $\tau_{t, x}^{\xi}: T_{\gamma_{x}(t)} \widetilde{M} \rightarrow T_{\gamma_{x}(0)} \widetilde{M}$ the parallel transport in $\tilde{M}$ along the curve $\gamma_{x}:=\tilde{\gamma}_{x}^{\xi}$. To compute $D_{\tilde{f}}(\xi)(s)$ in general, one 
needs to consider the expression $\frac{1}{2} \tau_{t, x}^{\xi}\left(d \tilde{f}_{t}(s)+\tilde{J} d \tilde{f}_{t}(j s)\right)$ and take its derivative with respect to $\mathrm{t}$ at $t=0$ :

$$
D_{\tilde{f}}(\xi)(s)=\frac{1}{2} \frac{\partial}{\partial t}\left(\tau_{t, x}^{\xi}\left(d \tilde{f}_{t}(s)+\tilde{J} d \tilde{f}_{t}(j s)\right)\right)_{\left.\right|_{t=0}}
$$

We define Const to be the subspace of $C^{\infty}$ (triv) made out of constant sections. For the proof of the theorem, we are particularly interested in computing $D_{1 \text {,hor }}$ and the restriction of $D_{1 \text {,vert }}$ to Const.

To simplify the notation, we denote by $x$ the coordinate on $\Sigma$ and write the points in $\mathbb{C}^{m} \times M$ as $\left(z_{1}, \ldots, z_{m}, y\right)$, where $z_{1}=w_{1}+i v_{1}$ and so on. For simplicity we denote coordinate vector fields in Const by $\partial_{w_{k}}:=\partial / \partial w_{k}$ and so on. Since we are going to work with an arbitrary choice of $w_{k}$ and $v_{k}$ we will refer to them simply as $\partial_{w}$, unless we need to be more specific.

Lemma 4.4. Let the notation be as above.

(i) $D_{2, \text { hor }}=0$.

(ii) $D_{2, \text { vert }}=D_{f}$.

(iii) $D_{1, \text { hor }}(\xi)=\bar{\partial}_{\mathbb{C}^{m}}(\xi)$ for all $\xi \in C^{\infty}$ (triv), where $\bar{\partial}_{\mathbb{C}^{m}}$ is the delbar operator in $\mathbb{C}^{m}$.

(iv) $\left(D_{1, \text { vert }}\right)\left(\partial_{z}\right)(s)=\frac{1}{2}(\partial / \partial z)(J(z))_{\mid z=0}(d f(j s))$ for $\partial_{z}$ a coordinate vector field in Const $\subset C^{\infty}$ (triv).

Proof. Since $\tilde{f}=f \circ i \subset\{0\} \times M$ we can naturally view any $\xi \in C^{\infty}\left(f^{*} T M\right)$ as an element in $C^{\infty}\left(\tilde{f}^{*} T \widetilde{M}\right)$ with values in the vertical direction tangent to $\{0\} \times M$. We have

$$
\widetilde{F}_{\xi}(t, x)=\exp _{\tilde{f}(x)}^{\widetilde{\nabla}}(t \xi)=\exp _{f(x)}^{\nabla}(t \xi),
$$

with $\operatorname{im} \widetilde{F} \subset\{0\} \times M$. This implies that the $d \tilde{f}_{t}(s)$ are also vertical vector fields supported in $\{0\} \times M$ and, since $\tilde{J}$ keeps $T(\{0\} \times M)$ invariant, we have as well that the $\tilde{J} d \tilde{f}_{t}(j s)$ are vertical vector fields in $\{0\} \times M$. Similarly, $\widetilde{F}_{\xi}^{*}(\partial / \partial t)$ is a vertical section in $T \widetilde{M}$ supported in $\{0\} \times M$ and parallel transport along $\tilde{f}(x)$ with respect to $\widetilde{\nabla}$ is the same as parallel transport with respect to $\nabla$.

A direct application of (22) is that

$$
\left(D_{\tilde{f}} \xi\right)(s)=\frac{1}{2} \frac{\partial}{\partial t}\left(\tau_{t, x}^{\xi} d \tilde{f}_{t}(s)+\tau_{t, x}^{\xi} \tilde{J} d f_{t}(j s)\right)_{\left.\right|_{t=0}}=\left(D_{f} \xi\right)(s),
$$

which proves (i). Relation (ii) follows immediately from the formula above, taking into account that $D_{\tilde{f}} \xi=D_{2 \text {,vert }}(\xi)$ and that $\left.\operatorname{im} D_{\tilde{f}}\right|_{C^{\infty} f * T M} \subset \Omega_{J}^{0,1}\left(\Sigma, f^{*} T M\right)$.

For the proofs of (iii) and (iv) we now consider $\xi \in C^{\infty}$ (triv). We can assume 
$\xi=\phi(x) \partial_{w}$, where $\phi: \Sigma \rightarrow \mathbb{C}^{m}$. In this situation, $\widetilde{F}_{\xi}(t, x)=\exp _{\tilde{f}(x)} \widetilde{\widetilde{v}}_{f}\left(t \partial_{w}\right)=$ $(\phi(x) t, 0, \ldots, 0, f(x))$. Thus the paths $\gamma_{x}$ are straight lines in $\mathbb{C}^{n} \times f(x) \subset \tilde{M}$ and parallel transport $\tau_{t, x}: T_{(t, f(x))} \widetilde{M} \rightarrow T_{0, f(x))} \widetilde{M}$ along $\gamma_{x}$ is the identity. We are also going to consider the coordinates $x \in \Sigma$ of the type $x=x_{1}+i x_{2}$, and do our computations for $s=\partial_{x_{1}}$.

If $\tilde{J}(t)$ is the almost complex structure at $\tilde{\gamma}_{x}^{\xi}(t)$, then $\tilde{J}(t)$ has the form

$$
\left(\begin{array}{ll}
A_{t} & 0 \\
B_{t} & J_{t}
\end{array}\right)
$$

with respect to the product structure $\mathbb{C}^{m} \times M$. Moreover along $\pi^{-1}(0)$ we have

$$
\tilde{J}(0)=\left(\begin{array}{cc}
J_{\mathbb{C}^{m}} & 0 \\
0 & J_{t}
\end{array}\right)
$$

Therefore $(\partial / \partial t) \tilde{J}(t)$ preserves the fibers, as does $\tilde{J}(t)$. Moreover, along $\{0\} \times M$, $\tilde{J}(0)$ preserves the splitting into $T M$ and $H$. As we have seen, parallel transport along $\tilde{\gamma}_{x}^{\xi}(t)$ is the identity.

Considering local coordinates $x=x_{1}+i x_{2}$ on $\Sigma$ and taking $s=\partial_{x_{1}}$, we have

$$
\begin{aligned}
D_{1, \text { hor }}\left(\phi \partial_{w}\right)\left(\partial_{x_{1}}\right)= & \frac{1}{2} \operatorname{proj}_{H} \frac{\partial}{\partial t}\left(\tau_{t, x}^{\xi} d \tilde{f}_{t}\left(\partial_{x_{1}}\right)+\frac{1}{2} \tau_{t, x}^{\xi} \tilde{J} d \tilde{f}_{t}\left(j \partial_{x_{1}}\right)\right)_{\left.\right|_{t=0}} \\
= & \frac{1}{2} \operatorname{proj}_{H} \frac{\partial}{\partial t}\left(d \tilde{f}_{t}\left(\partial_{x_{1}}\right)+\frac{1}{2} \tilde{J}_{d} \tilde{f}_{t}\left(\partial_{x_{2}}\right)\right)_{\left.\right|_{t=0}} \\
= & \frac{1}{2} \frac{\partial}{\partial t}\left(\partial_{x_{1}}(\phi(x)) t, 0, \ldots, 0\right)_{\left.\right|_{t=0}}+\frac{1}{2} \operatorname{proj}_{H} \frac{\partial}{\partial t}\left(\tilde{J}_{t}\right)_{\mid t=0} d f\left(\partial_{x_{2}}\right) \\
& \quad+\frac{1}{2} \operatorname{proj}_{H} \tilde{J}_{0} \frac{\partial}{\partial t}\left(\partial_{x_{2}}(\phi(x)) t, 0, \ldots, 0, d f(x)\right)_{\mid t=0},
\end{aligned}
$$

where, as mentioned before, $\phi: \Sigma \rightarrow \mathbb{C}^{m}$. But the middle term on the right-hand side vanishes because $d f\left(\partial_{x_{2}}\right)$ is a vertical vector and $\partial / \partial t \tilde{J}$ preserves fibers, so $(\partial / \partial t)\left(\tilde{J}_{t}\right)_{\mid t=0} d f\left(\partial_{x_{2}}\right)$ is also a vertical vector. Then

$$
D_{1, \operatorname{hor}}\left(\phi \partial_{w}\right)\left(\partial_{x_{1}}\right)=\frac{1}{2} \partial_{x_{1}} \phi(x)+\frac{1}{2} J_{\mathbb{C}^{m}}\left(\partial_{x_{2}}\right) \phi(x)
$$

For the last expression we have to use that along $\pi^{-1}(0), \tilde{J}_{0}$ preserves the horizontal space $H$, so $\operatorname{proj}_{H} \circ \tilde{J}_{0}=\tilde{J}_{\mathbb{C}^{m}} \circ \operatorname{proj}_{H}$. Therefore, the conclusion follows that $D_{1, \text { hor }}=\bar{\partial}_{\mathbb{C}^{m}}$.

To prove point (iv) of the theorem we now need to consider $\xi=\partial_{w} \in$ Const. Under this assumption we have $\tau_{t, x}^{\partial_{w}} d \tilde{f}_{t}=d f_{0}$. Thus

$$
\frac{\partial}{\partial t} \tau_{t, x}^{\partial_{w}} d \tilde{f}_{t}(s)=0
$$


As before, $s$ is a just a section in $T \Sigma$. Then

$$
\begin{aligned}
& D_{1, \text { vert }}\left(\partial_{w}\right)(s)=\frac{1}{2} \operatorname{proj}_{V} \frac{\partial}{\partial t}\left(\tau_{t, x}^{\partial w} d \tilde{f}_{t}(s)+\frac{1}{2} \tau_{t, x}^{\partial w} \tilde{J} d \tilde{f}_{t}(j s)\right)_{\left.\right|_{t=0}} \\
& =\frac{1}{2} \operatorname{proj}_{V} \frac{\partial}{\partial t}\left(\tau_{t, x}^{\partial w} d \tilde{f}_{t}(s)\right)_{\left.\right|_{t=0}}+\frac{1}{2} \operatorname{proj}_{V} \frac{\partial}{\partial t}\left(\tau_{t, x}^{\partial w} \tilde{J}\left(\tau_{t, x}^{\partial w}\right)^{-1}\right)_{\left.\right|_{t=0}} \cdot d f(j s) \\
& +\frac{1}{2} \operatorname{proj}_{V} \tilde{J}_{0}\left(\frac{\partial}{\partial t} \tau_{t, x}^{\partial w} d \tilde{f}_{t}(j s)\right)_{\mid t=0} \\
& =\frac{1}{2} \operatorname{proj}_{V}\left(\widetilde{\nabla}_{\partial_{w}} \tilde{J}\right) d f(j s),
\end{aligned}
$$

where we denote by $\operatorname{proj}_{V}$ the projection onto the fibers. Recall that $(\partial / \partial t) \tilde{J}$ takes vertical vector fields into vertical vector fields. Therefore

$$
\frac{1}{2} \operatorname{proj}_{V} \widetilde{\nabla}_{\partial_{w}} \tilde{J} d f(j s)=\frac{1}{2} \frac{\partial J(z)}{\partial w}(d f(j s)),
$$

precisely because $d f(j s)$ is a vertical vector field and the covariant derivative along horizontal vector fields was chosen to be the standard connection in $\mathbb{C}^{m}$. Applying the same reasoning to $i \partial_{v}$, we see that

$$
\left(D_{1, \mathrm{vert}}\right)\left(\partial_{z}\right)(s)=\frac{1}{2} \frac{\partial}{\partial z}(J(z))_{\mid z=0}(d f(j s)) .
$$

It is worth pointing out that $(\partial / \partial z)(J(z))_{\mid z=0}=d \psi_{0}^{*}(\partial / \partial z)$.

Proof of Theorem 2.9. Direct implication: Using Lemma 4.4(v) we get the commutativity of the diagram

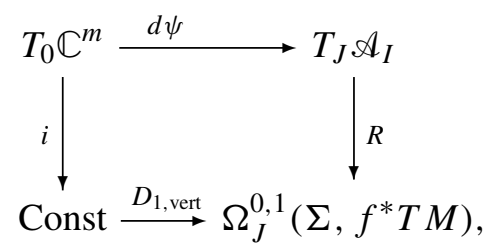

where $i: T_{0} \mathbb{C}^{n} \rightarrow$ Const $\subset C^{\infty}$ (triv) is the natural identification map and $\psi$ is the morphism from the parameter space to the space of almost complex structures. $R$ is, as mentioned before, given by $R(Y)=\frac{1}{2} Y \circ d f \circ j$.

Since $D_{\tilde{f}}$ is surjective by hypothesis, this means that $D_{1} \oplus D_{2}$ is surjective. We therefore conclude, by Lemma 4.4(i,ii), that

$$
D_{1}=\left(D_{1, \text { vert }}, D_{1, \text { hor }}\right): C^{\infty}(\text { triv }) \longrightarrow \operatorname{coker} D_{f} \oplus \Omega_{\tilde{J}}^{0,1}(\Sigma, H)
$$

is surjective. Since the kernel of the $\bar{\partial}_{\mathbb{C}^{m}}$ operator on $\mathbb{C}^{m}$ consists precisely of constant sections, Lemma 4.4(iii) implies that $D_{1 \text {,hor }}^{-1}(0)=$ Const. Therefore the operator $\left(D_{1, \text { vert }}\right)_{\mid \text {Const }}$ : Const $\rightarrow \operatorname{coker} D_{f}$ is surjective. But this will imply that

$$
\left(D_{1, \text { vert }}\right)_{\text {Const }} \circ i: T_{0} \mathbb{C}^{m} \rightarrow \operatorname{coker} D_{f}
$$

is surjective. 
As we saw in the proof of Proposition 2.8, $R$ induces an isomorphism

$$
\tilde{R}: \widetilde{\operatorname{coker} d} \Pi \longrightarrow \operatorname{coker} D_{2},
$$

and moreover the diagram (24) will be still commutative if we restrict $d \psi$ and $D_{1, \text { vert }}$ to coker $d \Pi$ and coker $D_{2}$ respectively. Therefore $d \psi: T_{0} \mathbb{C}^{n} \rightarrow \operatorname{coker} d \Pi$ is surjective. By Proposition 2.8, this yields parametric regularity.

For the inverse implication, notice that $D_{1, \text { hor }}$ will cover the space $\Omega_{\tilde{J}}^{0,1}(\Sigma, H)$ when $\Sigma=S^{2}$, because $D_{1, \text { hor }}=\bar{\partial}_{\mathbb{C}^{m}}$ in this case. By hypothesis, $d \psi: T_{0} \mathbb{C}^{n} \rightarrow$ coker $d \Pi$ is surjective and the preceding observation implies that

$$
D_{1}=\left(D_{1, \text { vert }}, D_{1, \text { hor }}\right): C^{\infty}(\text { triv }) \longrightarrow \operatorname{coker} D_{f} \oplus \Omega_{\tilde{J}}^{0,1}(\Sigma, H)
$$

is also surjective. Therefore $D_{\tilde{f}}$ is a surjective operator.

\section{Acknowledgments}

This work is part of the author's doctoral research at SUNY Stony Brook. The author thanks her advisor, Dusa McDuff, for her suggestions, advice and comments on earlier drafts.

\section{References}

[Abreu 1998] M. Abreu, “Topology of symplectomorphism groups of $S^{2} \times S^{2}$,, Invent. Math. 131:1 (1998), 1-23. MR 99k:57065 Zbl 0902.53025

[Abreu and McDuff 2000] M. Abreu and D. McDuff, "Topology of symplectomorphism groups of rational ruled surfaces", J. Amer. Math. Soc. 13:4 (2000), 971-1009. MR 2001k:57035 ZBL 0965. 57031

[Aebischer et al. 1994] B. Aebischer, M. Borer, M. Kälin, C. Leuenberger, and H. M. Reimann, Symplectic geometry, Progress in Mathematics 124, Birkhäuser, Basel, 1994. MR 96a:58082 Zbl 0932.53002

[Bryan and Leung 2000] J. Bryan and N. C. Leung, "The enumerative geometry of $K 3$ surfaces and modular forms", J. Amer. Math. Soc. 13:2 (2000), 371-410. MR 2001i:14071 Zbl 0963.14031

[Fukaya and Ono 1999] K. Fukaya and K. Ono, "Arnold conjecture and Gromov-Witten invariant", Topology 38:5 (1999), 933-1048. MR 2000j:53116 Zbl 0946.53047

[Kronheimer 1998] P. Kronheimer, "Some non-trivial families of symplectic structures", preprint, Harvard University, 1998, Available at http://www.math.harvard.edu/ kronheim/diffsymp.pdf.

[Lê and Ono 2001] H. V. Lê and K. Ono, "Parameterized Gromov-Witten invariant and topology of symplectomorphism groups", preprint 28/2001, Max Planck Institute for Mathematics in the Sciences, 2001, Available at http://www.mis.mpg.de/preprints/2001/prepr2801-abstr.html.

[Li and Tian 1998] J. Li and G. Tian, "Virtual moduli cycles and Gromov-Witten invariants of general symplectic manifolds", pp. 47-83 in Topics in symplectic 4-manifolds (Irvine, CA, 1996), edited by R. J. Stern, Internat. Press, Cambridge, MA, 1998. MR 2000d:53137 Zbl 0978.53136

[McDuff 2000] D. McDuff, “Almost complex structures on $S^{2} \times S^{2}$ ”, Duke Math. J. 101:1 (2000), 135-177. MR 2001h:53126 Zbl 0974.53020 
[McDuff 2001] D. McDuff, "Symplectomorphism groups and almost complex structures", pp. 527556 in Essays on geometry and related topics, vol. 2, Monogr. Enseign. Math. 38, Enseignement Math., Geneva, 2001. MR 2003i:57042 Zbl 1010.53064

[McDuff and Salamon 1994] D. McDuff and D. Salamon, J-holomorphic curves and quantum cohomology, vol. 6, University Lecture Series, American Mathematical Society, Providence, RI, 1994. MR 95g:58026 Zbl 0809.53002

[Ruan 1999] Y. Ruan, "Virtual neighborhoods and pseudo-holomorphic curves", Turkish J. Math. 23:1 (1999), 161-231. MR 2002b:53138 Zbl 0967.53055

[Seidel 1997] P. Seidel, Floer homology and the symplectic isotopy problem, Ph.D. thesis, Oxford University, 1997.

[Seidel 1999] P. Seidel, "On the group of symplectic automorphisms of $\mathbf{C P}^{\mathbf{m}} \times \mathbf{C P}^{\mathbf{n}}$ ", pp. 237-250 in Northern California Symplectic Geometry Seminar, edited by Y. Eliashberg et al., Amer. Math. Soc. Transl. Ser. 2 196, Amer. Math. Soc., Providence, RI, 1999. MR 2000m:53124 Zbl 0954.58009

[Smale 1965] S. Smale, "An infinite dimensional version of Sard's theorem”, Amer. J. Math. 87 (1965), 861-866. MR 32 \#3067 Zbl 0143.35301

Received January 29, 2003.

Olguța Bușe

DEPARTMENT OF MATHEMATICS

A-320 Wells Hall

Michigan State University

EAST LANSING, MI 48824

UNited STATES

buse@math.msu.edu 